\title{
Microstructural Factors of Strain Delocalization in Model Metallic Glass Matrix Composites
}

Thomas James Hardin

Brigham Young University - Provo

Follow this and additional works at: https://scholarsarchive.byu.edu/etd

Part of the Mechanical Engineering Commons

\section{BYU ScholarsArchive Citation}

Hardin, Thomas James, "Microstructural Factors of Strain Delocalization in Model Metallic Glass Matrix Composites" (2014). Theses and Dissertations. 4079.

https://scholarsarchive. byu.edu/etd/4079

This Thesis is brought to you for free and open access by BYU ScholarsArchive. It has been accepted for inclusion in Theses and Dissertations by an authorized administrator of BYU ScholarsArchive. For more information, please contact scholarsarchive@byu.edu, ellen_amatangelo@byu.edu. 


\title{
Microstructural Factors of Strain Delocalization \\ in Model Metallic Glass Matrix Composites
}

Thomas J. Hardin

\author{
A thesis submitted to the faculty of \\ Brigham Young University \\ in partial fulfillment of the requirements for the degree of \\ Master of Science
}

Eric R. Homer, Chair

David T. Fullwood

Michael Miles

\section{Department of Mechanical Engineering \\ Brigham Young University}

June 2014

Copyright (C) 2014 Thomas J. Hardin

All Rights Reserved 


\author{
ABSTRACT \\ Microstructural Factors of Strain Delocalization \\ in Model Metallic Glass Matrix Composites \\ Thomas J. Hardin \\ Department of Mechanical Engineering, BYU \\ Master of Science
}

Metallic glass matrix composites have enormous potential stemming from the interplay between crystalline and amorphous phases. This work models such a composite using shear transformation zone dynamics (a modified kinetic Monte Carlo method) for the amorphous phase, and a local Taylor dislocation model for the crystalline phase. An N-factorial experiment using the model is presented examining the effects of crystalline volume fraction, microstructure length scale, and yield stress of the crystalline phase. Each replicate is analyzed for maximum stress, maximum strain, strain energy dissipation, strain localization, and strain partitioning between phases. Regression analysis is used to identify statistically-significant trends in the data. The experiment shows that strain delocalization and the consequent ductility are facilitated by a crystalline phase with a substantially lower yield stress than that of the amorphous matrix. It also shows that increasing crystalline volume fraction alone is insufficient to promote strain delocalization in the case of a crystalline phase with high relative yield stress, and that a lower yield stress for the crystalline phase implies lower maximum stresses supported by the composite. Therefore designers must balance the need for ductility and delocalization against the composite yield stress by finding an optimal combination of volume fraction and crystalline mechanical properties. This work

provides continuous functional forms for the relationships between these properties to aid in that optimization process.

Keywords: metallic glass, amorphous, composite, STZ dynamics 


\section{ACKNOWLEDGMENTS}

I don't wish to seem ungrateful

To those whose influences fateful

Guided me along my way

Gave good advice from day to day

Among acknowledged blessings mine:

Great mentors are a gift divine.

This work was funded by a NASA Space Technology Research Fellowship, Grant \# NNX13AL43H. 


\section{TABLE OF CONTENTS}

LIST OF TABLES $\ldots \ldots \ldots \ldots \ldots \ldots \ldots \ldots \ldots \ldots \ldots$ vi

LIST OF FIGURES $\ldots \ldots \ldots \ldots \ldots \ldots \ldots \ldots$ vii

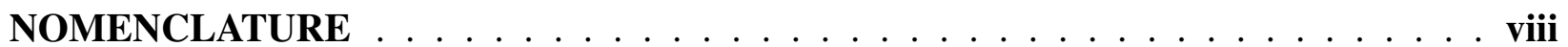

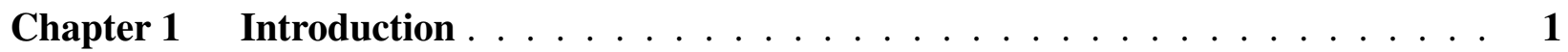

Chapter 2 Model $\ldots \ldots \ldots \ldots \ldots \ldots \ldots \ldots \ldots$

Chapter 3 Method: Full $3 \times 2^{3}$ Factorial Experiment $\ldots \ldots \ldots \ldots$

3.1 Independent Factors . . . . . . . . . . . . . . . . . . . . . . 8

3.1 .1 Crystal Volume Fraction $(V) \ldots \ldots \ldots \ldots$

3.1.2 Crystalline Inclusion Length Scale $(L) \ldots \ldots \ldots \ldots$

3.1 .3 Yield Stress Ratio $(R) \quad \ldots \ldots \ldots$

3.2 Design of Experiment . . . . . . . . . . . . . . . . . . . . . . . . . 10

3.3 Dependent (Response) Variables . . . . . . . . . . . . . . . . . . . . . . 10

3.3.1 Maximum Stress $\left(\sigma_{\max }\right) \ldots \ldots \ldots \ldots$

3.3.2 Tensile Strain to Failure $\left(\varepsilon_{\max }\right) \ldots \ldots \ldots \ldots \ldots$

3.3.3 Strain Energy to Failure $(E) \ldots \ldots \ldots \ldots \ldots$

3.3.4 Localization Index $(\Gamma) \ldots \ldots \ldots \ldots$

3.3.5 Two-Point Statistic Localization $\left(L_{2}\right) \quad \ldots \ldots \ldots \ldots \ldots \ldots$

3.3.6 Uniaxiality of Strain Fields $(U) \quad \ldots \ldots \ldots$

3.3.7 Phase Strain Average Ratio $\left(R_{\varepsilon}\right) \ldots \ldots \ldots \ldots$

3.4 Summary of Variables . . . . . . . . . . . . . . . . . . . . . . . . . . . . 14

Chapter 4 Results . . . . . . . . . . . . . . . . . . . . . . . . . . 15

4.1 Regression Analysis of Results . . . . . . . . . . . . . . . . . . . 15

Chapter 5 Discussion . . . . . . . . . . . . . . . . . . 21

5.1 Implications for Design . . . . . . . . . . . . . . . . . 23

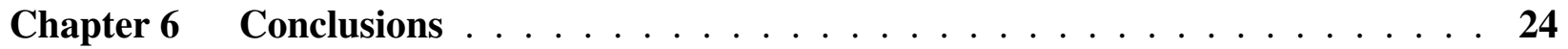

6.1 Looking Forward $\ldots \ldots \ldots \ldots \ldots \ldots$

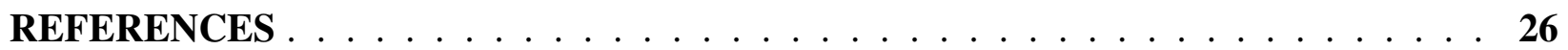

Appendix A Coded Variables and Preconditioned Results $\ldots \ldots \ldots \ldots$

Appendix B Regression Details $\ldots \ldots \ldots \ldots \ldots \ldots \ldots$

B.1 Maximum Stress $\left(\sigma_{\max }\right) \ldots \ldots \ldots \ldots \ldots$

B.2 Tensile Strain to Failure $\left(\varepsilon_{\max }\right) \ldots \ldots \ldots \ldots \ldots \ldots$ 
B.3 Strain Energy to Failure $(E) \quad \ldots \ldots \ldots \ldots \ldots$

B.4 Localization Index $(\Gamma) \ldots \ldots \ldots \ldots$

B.5 Two-Point Statistic Localization $\left(L_{2}\right) \ldots \ldots \ldots \ldots \ldots$

B.6 Uniaxiality $(U) \ldots \ldots \ldots \ldots \ldots$

B.7 Phase Strain Average Ratio $\left(R_{\varepsilon}\right) \ldots \ldots \ldots \ldots$

Appendix C Strain Fields and Two-Point Correlations for All Runs . . . . . . . . . . 38

Appendix D Regression Analysis Mathematica Code . . . . . . . . . . . . . . 47 


\section{LIST OF TABLES}

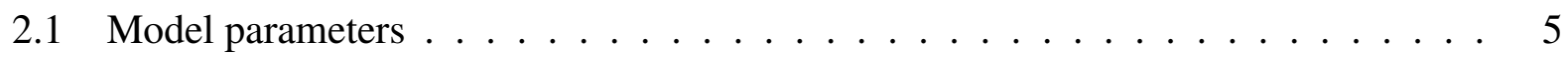

3.1 Experimental factors and levels . . . . . . . . . . . . . . . 9

4.1 Experimental results . . . . . . . . . . . . . . . . . . . 17

4.2 Summary of regression coefficients . . . . . . . . . . . . . . . . 19

A.1 Coded and preconditioned results . . . . . . . . . . . . . 31

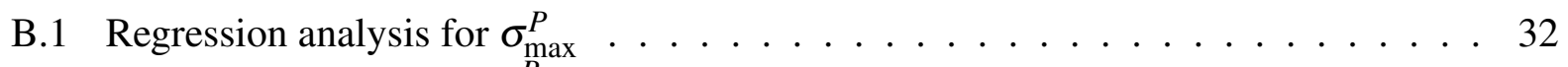

B.2 Regression analysis for $\varepsilon_{\max }^{P} \ldots \ldots \ldots \ldots \ldots \ldots$

B.3 Regression analysis for $E^{P} \ldots \ldots \ldots \ldots \ldots \ldots$

B.4 Regression analysis for $\Gamma^{P} \ldots \ldots \ldots \ldots$

B.5 Regression analysis for $L_{2}^{P} \ldots \ldots \ldots \ldots \ldots$

B.6 Regression analysis for $R_{\varepsilon}^{P} \ldots \ldots \ldots \ldots \ldots$ 


\section{LIST OF FIGURES}

2.1 Amorphous and crystalline stress-strain curves . . . . . . . . . . . . 5

2.2 Schematic of model implementation in finite elements . . . . . . . . . . . . . 7

3.1 Schematic of two-point localization analysis . . . . . . . . . . . . 13

4.1 Graphical results . . . . . . . . . . . . . . . . . . . . 16

4.2 Shear band fragmentation . . . . . . . . . . . . . . . . . 17

4.3 Regression algorithm schematic . . . . . . . . . . . . . . . . . 18

4.4 Graphical regression results . . . . . . . . . . . . . . . . . 20

5.1 Interaction modes between shear bands and inclusions $\ldots \ldots 22$

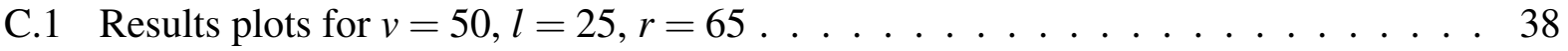

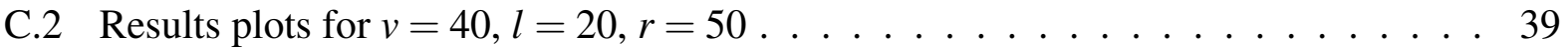

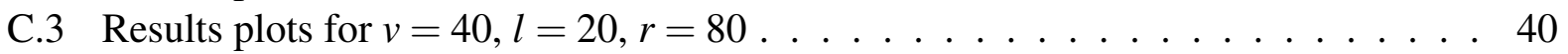

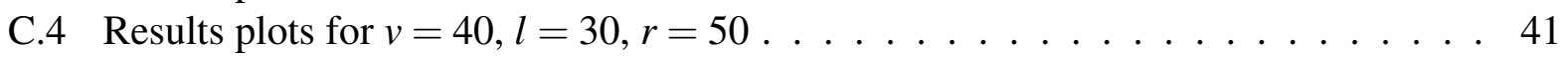

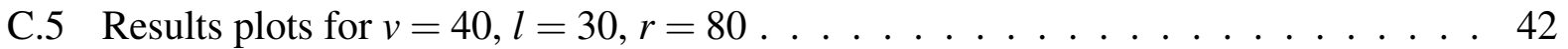

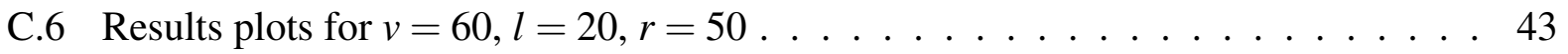

C.7 Results plots for $v=60, l=20, r=80 \ldots \ldots \ldots \ldots \ldots \ldots \ldots \ldots$

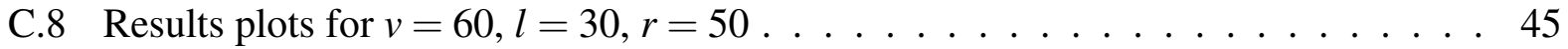

C.9 Results plots for $v=60, l=30, r=80 \ldots \ldots \ldots \ldots \ldots$ 


\section{NOMENCLATURE}

$\alpha \quad$ Statistical significance level, set to 0.01 in this study

$a \quad$ An empirical constant in the crystalline plasticity model

ANOVA Analysis of variance

$b \quad$ Burger's vector length

BMG Bulk metallic glass

$\varepsilon \quad$ Strain

$\varepsilon_{\max } \quad$ Tensile strain to failure

$E \quad$ Young's modulus, Strain energy to failure

$\Delta F \quad$ Intrinsic barrier height for STZ transition

$\mathscr{F} \quad$ Fourier transform

$\gamma_{0} \quad$ STZ shear strain increment

$\Gamma \quad$ Localization index

$k \quad$ Boltzmann constant

kMC Kinetic Monte Carlo

$L, l \quad$ Length scale of crystalline inclusions

$L_{2} \quad$ Two-point statistic localization

$\mu \quad$ Shear modulus

MGMC Metallic glass matrix composite

$n \quad$ Hardening coefficient in the crystalline plasticity model

$P \quad$ Spectral norm of plastic strain field, Probability of statistical results being entirely due to chance

$Q \quad$ A right-preconditioning matrix

$R, r \quad$ Ratio of yield stresses between crystalline and amorphous phases

$R_{\varepsilon} \quad$ PHase strain average ratio

$R^{2} \quad$ A goodness-of-fit metric for a regression model

$\sigma \quad$ Stress

$\sigma_{\max } \quad$ Maximum stress

$\dot{s} \quad$ STZ activation rate

STZ Shear transformaton zone

$T \quad$ Temperature, or Two-point localization field

$\tau \quad$ Local stress state of STZ

$U \quad$ Uniaxiality of strain field

$v_{0} \quad$ Attempt frequency

$v \quad$ Poisson's ratio

$V, v \quad$ Volume fraction of crystalline phase

$\Omega_{0} \quad$ Volume of an STZ 


\section{CHAPTER 1. INTRODUCTION}

Metallic glass matrix composites (MGMC) have demonstrated enormous potential for improved ductility and toughness over traditional bulk metallic glasses (BMG). Some MGMCs even exhibit toughness comparable to that of aluminum or steel alloys $[2,3]$ while retaining impressive strength and stiffness [4]. Of fundamental interest in the design of these MGMCs is the role played by the various microstructural characteristics of the two contributing phases that enable further optimization of these composites.

The crystalline inclusions in MGMCs are needed to improve the plasticity of BMGs because monolithic metallic glasses (amorphous metals) typically exhibit catastrophic failure by shear banding upon yield. This extreme response results from the absence of a crystal lattice, which precludes the plasticity mechanisms found in traditional crystalline materials. Plasticity in amorphous metals occurs by incremental localized shear events called shear transformation zones (STZ) [5]. These zones involve the collective rearrangement of several dozen atoms in response to an applied shear stress. These thermally activated STZs typically have volumes on the order of $10^{-27} \mathrm{~m}^{3}$ and shear over timescales of $10^{-12} \mathrm{~s}$ [6-11]. STZs are energetically much more costly than dislocations or twinning and differ in that they leave behind local structural changes involving increased free volume [6]. These structural changes and the stress fields in the vicinity of an STZ bias the energy landscape in favor of further STZ activation nearby; consequently, a chain of subsequent STZs activate in this "softer" region, leading to the sudden, catastrophic failure mentioned earlier [12]. As a result of this strain-softening behavior and accompanying catastrophic brittle failure mode, monolithic metallic glasses have struggled to find engineering application leveraging their extraordinarily high strength-to-weight ratio.

The introduction of a second, crystalline phase into the amorphous matrix (to form an MGMC) breaks up shear band events that would otherwise cause failure. This second phase is introduced either in the form of intrinsic crystalline dendrites which nucleate and grow in certain 
alloys under specific processing conditions $[13,14]$, or by addition of extrinsic metal whiskers or particles [15-17].

Recent experimental work has focused on optimizing MGMCs for various loading conditions; behavior under dynamic loading $[18,19]$ and ductility under tensile loading have been of particular interest [4, 20-25]. Other experiments have studied how processing-specifically cold rolling — enhances ductility of MGMCs [23, 24]. One recent development has demonstrated strain-hardening behavior in MGMC alloys whose crystalline phase exhibits a martensitic transformation [26-29].

Efforts to examine the microstructural factors governing MGMC behavior in a systematic manner have yielded insight towards optimizing such composites. After chemistry, volume fraction of the crystalline phase is the most-examined MGMC design variable [3,30-35]; it seems that increasing crystalline volume fraction tends to stabilize plasticity and delocalize strain, but to decrease the macroscopic strength of the composite. Length scale of the dendritic phase has also been investigated at length $[3,4,14,22,30,34,36]$ in an effort to find an optimum balance of ductility and strength. Finally, a very few experiments have explicitly examined the effect of ductility or brittleness of the second phase of the MGMC [35]; the effectiveness of the second phase appears to be dependent on the ductility of that phase, and not merely on the inhomogeneity of the composite.

Computational models have also contributed to understanding of MGMC mechanics [11]. At the continuum level, a two-phase finite element model by Qiao [37] quantitatively describes macroscopic MGMC deformation mechanics. The model is based on a five-step deformation regime which starts with pure elastic deformation, then adds plasticity in the crystalline phase, then goes through three stages where both phases yield, then the crystallites harden, and then finally the composite softens and fails. Other finite element approaches have focused on stress heterogeneity and consequent plastic mismatch between the two phases [38], and on the distribution of strain between the two phases at varying degrees of deformation [39].

On much smaller length and time scales, atomistic investigations have resolved many characteristics of STZs and bulk metallic glass behavior [11,40]. Molecular dynamics simulations of MGMCs have yielded insight into shear band behavior around very small crystallites of varying geometry, volume fraction, and arrangement [41]. Insight has also been obtained into shear band deflection in amorphous/amorphous composites [42]. 
Between the continuum and atomistic length and time scales, this paper develops a mesoscale MGMC model based on Homer and Schuh's STZ dynamics model [12,43-45]. It outlines an Nfactorial experiment designed to isolate the effects of volume fraction, length scale, and yield stress of the crystalline phase. The experiment design enables isolation of effects, helping designers to better access the best that both the amorphous matrix and the crystalline reinforcement have to offer. Seven metrics are developed to distill the experimental results, and regression analysis is used to identify statistically significant trends. Finally, these trends are discussed in the context of MGMC design. The principles and trends, and particularly the functional forms presented in this work will enable greater understanding and optimization of MGMCs, bringing them closer to widespread application. 


\section{CHAPTER 2. MODEL}

In the present model, the behavior of the amorphous matrix is given by the STZ dynamics model, which is based on stochastic activation of coarse-grained STZs [43]. An STZ activation represents an instantaneous, inelastic shearing of a cluster of atoms based on the cluster's local stress state. This is modeled by applying plastic strains to groups of elements in a finite element mesh that represent a potential STZ. After an STZ activates, finite-element analysis solves for the resultant stress and strain fields throughout the sample. The resulting stress and strain fields encourage shearing of further STZs (element clusters), and the process repeats. Selection of STZs to activate is controlled by a modified kinetic Monte Carlo (kMC) algorithm [46], based on the individual activation rates of an ensemble of STZs.

The activation rate $\dot{s}$ of an STZ is given by:

$$
\dot{s}=v_{0} \exp \left(-\frac{\Delta F-\frac{1}{2} \tau \gamma_{0} \Omega_{0}}{k T}\right)
$$

where $v_{0}$ is the attempt frequency (related to the Debye temperature), $\Delta F$ is the intrinsic barrier height of the STZ transition, $T$ is the temperature and $k$ is the Boltzmann constant. The activation rate is biased by the local stress state, $\tau$. Finally, $\gamma_{0}$ and $\Omega_{0}$ are the increment of shear strain applied to an STZ and the volume of an STZ, respectively. The values for the parameters used in this model are given in Table 2.1, and a characteristic stress-strain curve for the amorphous phase is shown in Figure 2.1. 
Table 2.1: Model parameters for amorphous and crystalline phases. Note that some parameters are set as levels for the experiment.

\begin{tabular}{lr}
\hline \hline Property & Amorphous Parameters \\
\hline Shear modulus & Symbol \& value \\
Poisson's ratio & $\mu=48.2 \mathrm{GPa}$ \\
Debye temperature & $\nu=0.352$ \\
Activation energy barrier & $372 \mathrm{~K}$ \\
STZ shear strain & $\Delta F=1.29 \mathrm{eV}$ \\
STZ volume & $\gamma_{0}=0.1$ \\
\hline & $\Omega_{0}=2.0 \mathrm{~nm}^{3}$ \\
\hline Property & Crystalline Parameters \\
\hline Young's modulus & Symbol \& value \\
Poisson's ratio & $E=106.3 \mathrm{GPa}$ \\
Yield stress & $v=0.33$ \\
Microstructure length & $\propto$ exp. level $r$ \\
Burger's vector & exp. level $l$ \\
Empirical constant & $b=2.858 \AA$ \\
Hardening coefficient & $a=0.1$ \\
\hline \hline
\end{tabular}

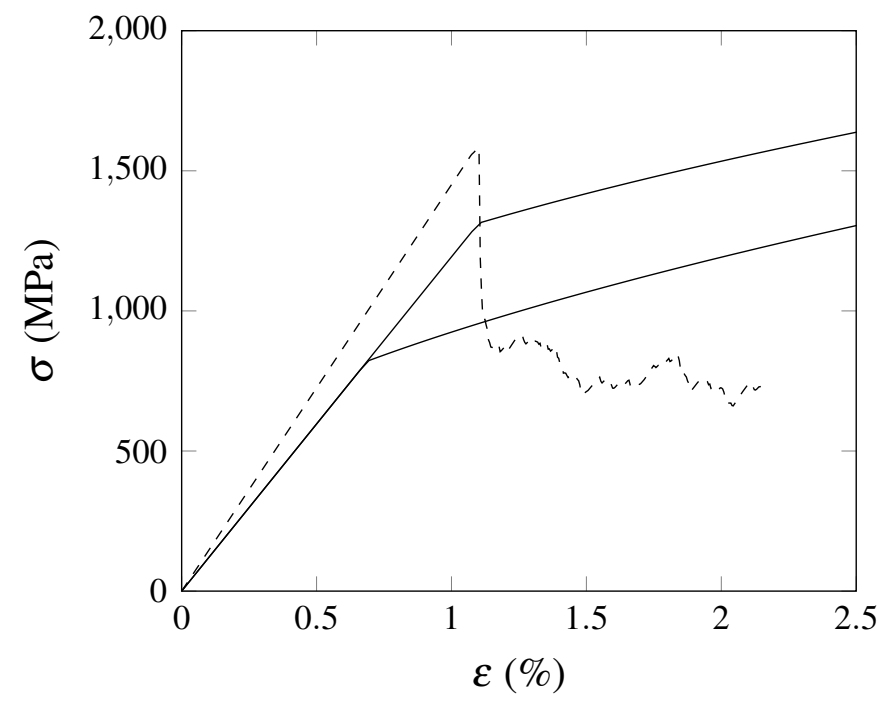

Figure 2.1: Stress-strain curves for amorphous matrix (the dashed line) and crystalline inclusions with two different yield points.

This paper reports extension of the STZ dynamics model to include a ductile phase, which is used to simulate the crystalline phase of an MGMC. This is accomplished by partitioning the mesh into the two phases, and applying the appropriate material or plasticity model to the elements 
of each phase. The finite element analysis solver evaluates the plastic deformation in the ductile phase in each $\mathrm{kMC}$ timestep. A maximum timestep of $0.01 \mathrm{~s}$ is enforced; see [46] for details.

Following the work of [37] and [22], the ductile plastic constitutive law is based on a Taylor dislocation model [47-49]. This is implemented as a UMAT subroutine in Abaqus. It is worth noting that the simulations in this work use microstructure length scales somewhat smaller than those for which the plasticity model has been validated; however, it still captures the requisite ductile behavior exhibited by MGMC microstructures. The plasticity model expresses the tensile stress-strain relation as follows:

$$
\sigma=\sigma_{r e f} \sqrt{\left(\sigma_{y} / E+\varepsilon^{p}\right)^{(2 n)}+L \bar{\eta}}
$$

where $\varepsilon^{p}$ is plastic strain, $E$ is Young's modulus, $\sigma_{y}$ is yield stress, $\sigma_{r e f}=E^{n} / \sigma_{y}^{n-1}, n$ is a hardening coefficient, $L=180 b\left(\frac{a \mu}{\sigma_{r e f}}\right)^{2}$ is an intrinsic material length with $\mu, b$, and $a$ being the shear modulus, Burgers vector length, and an empirical constant between 0.1 and 0.5 , and $\bar{\eta}$ is the average strain gradient, which is approximated by $\varepsilon^{p} / D$ where $D$ is a characteristic diameter of the crystalline phase microstructure. The quantities used in this experiment are shown in Table 2.1, and tensile stress-strain curves are shown in Figure 2.1 for two different yield strengths evaluated in this work.

The two models are merged in the finite-element model (see Fig. 2.2), which is partitioned into amorphous and crystalline elements. The crystalline inclusions are circular and are distributed pseudo-randomly across the sample (their positions were intially randomly seeded, then an optimization routine ensured that their positions were evenly distributed across the sample). The amorphous elements provide the set of potential STZs for the kMC algorithm described above. The crystalline elements' plasticity model is evaluated directly by Abaqus. The boundary between the two phases consists of coincident nodes-essentially a perfectly bound no-slip condition between the two. Time-steps are determined by kMC, and any stress or deformation loading can be applied in accordance with standard finite-element methods.

All samples in this paper are two-dimensional rectangles, 300nm in height and $100 \mathrm{~nm}$ in width. These are meshed into 212440 triangular elements of approximate size $0.53 \mathrm{~nm}$ on a side. While these dimensions are smaller than typical studies of MGMCs, the dimensions are limited by computational constraints. However, the trends observed do compare favorably to experiments on 
larger length scales. Samples are loaded in pure tension along the long (y) axis at a strain rate of $0.1 s^{-1}$.

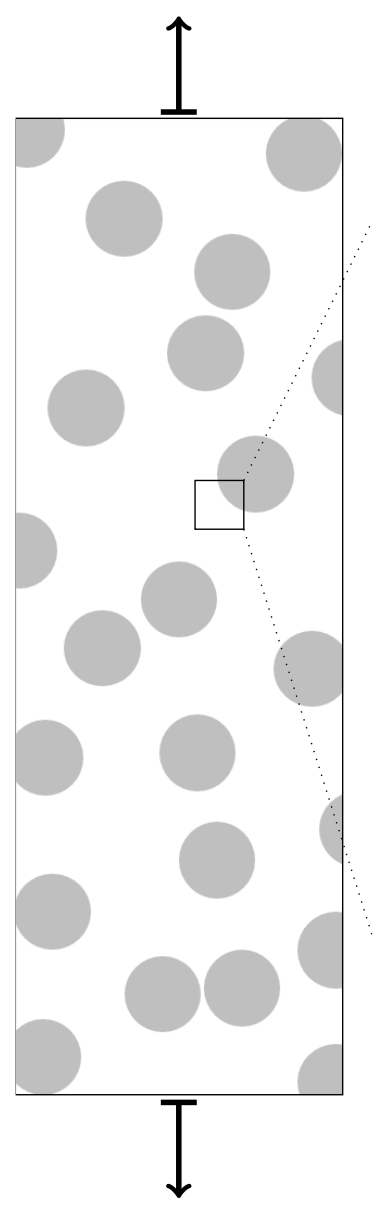

Boundary between phases consists of coincident nodes.

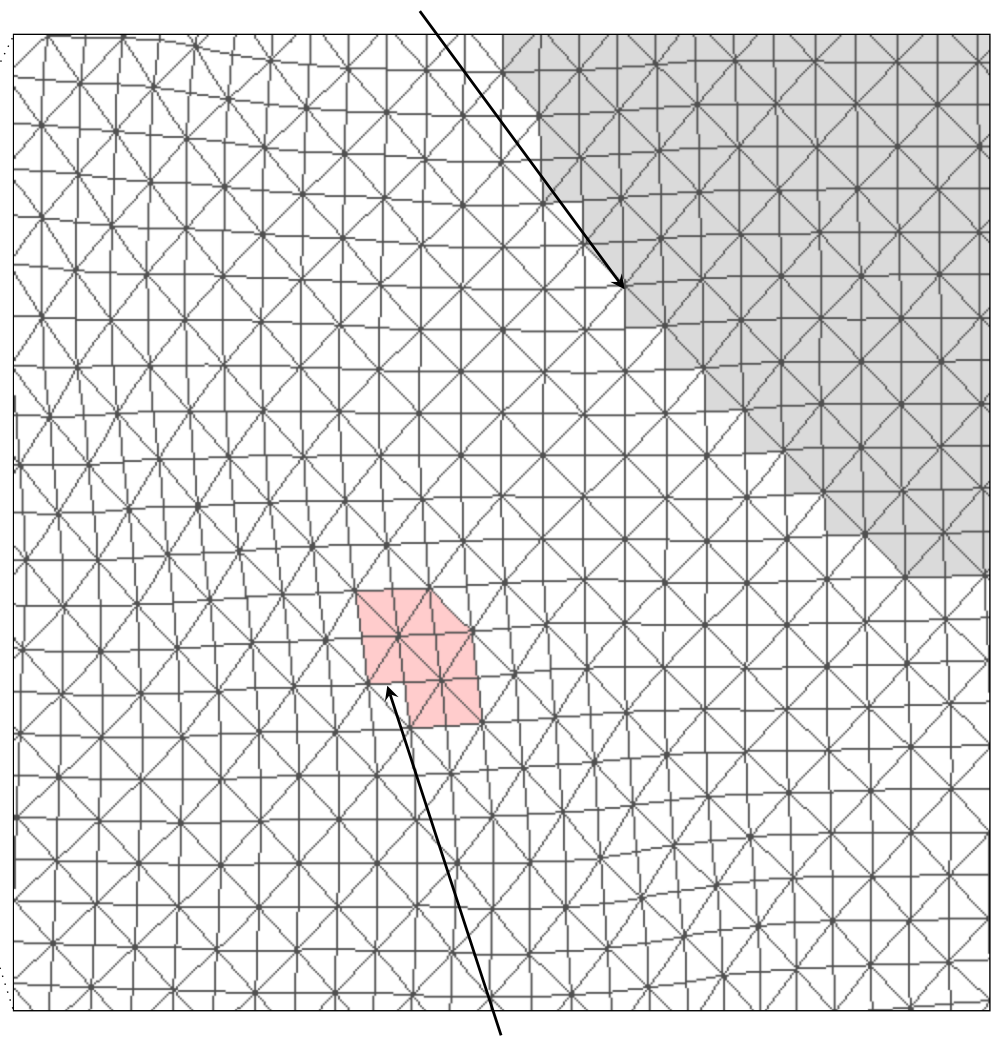

Each cluster of amorphous elements is a potential shear transformation zone.

Figure 2.2: Gray elements belong to the crystalline partition of the mesh, while white elements are amorphous in character. 


\section{CHAPTER 3. METHOD: FULL $3 \times 2^{3}$ FACTORIAL EXPERIMENT}

Factorial experiments study the effect of more than one factor simultaneously [50]. A few factors, three in this case, are selected for investigation, and then each of those factors are modulated between a few levels, two in this case. For simplicity, the levels are often assigned scaled values of -1 and +1 rather than using the actual experimental input quantities, and each treatment is set as a combination of levels for each factor. Often several replicates are repeated for each treatment, which reduces the statistical uncertainty of the results. By considering all possible combinations of the selected factors and levels as is done in a full-factorial experiment, effects and interactions between the factors can be isolated. Full-factorial designs of experiments can be powerful enough to show causation between independent and dependent variables.

Often a center point (treatment $(0,0,0))$ is also added to full-factorial designs, which improves statistical analysis and allows for estimates of nonlinearity in the data.

\subsection{Independent Factors}

The factors selected for this experiment are microstructure length scale of the crystalline inclusions, crystalline volume fraction, and ratio of yield stresses between the two phases; these are the principal variables of interest in most MGMC studies mentioned in the introduction of this paper. These independent factors and their levels are summarized in Table 3.1, and are described in detail in the following subsections. 
Table 3.1: Factors and levels for the $3 * 2^{3}+2 * 1$ factorial experiment.

Upper-case letters $(V L R)$ refer to level codes $-1,0$, or +1 , while lower-case letters $(v l r)$ refer to actual measured quantities.

\begin{tabular}{lrrrr}
\hline \hline Factor & Symbol & Low level & Mid level & High level \\
\hline Volume Fraction & $v(V)$ & $40 \%(-1)$ & $50 \%(0)$ & $60 \%(+1)$ \\
Length Scale & $l(L)$ & $20 \mathrm{~nm}(-1)$ & $25 \mathrm{~nm}(0)$ & $30 \mathrm{~nm}(+1)$ \\
Yield Stress Ratio & $r(R)$ & $50 \%(-1)$ & $65 \%(0)$ & $80 \%(+1)$ \\
\hline \hline
\end{tabular}

\subsubsection{Crystal Volume Fraction $(V)$}

One of the factors varied in this experiment is the volume fraction $(V)$ of the crystalline phase in an MGMC, since this has been shown to have a strong effect on composite behavior $[35,51]$. The level of $40 \%$ has been identified $[16,33]$ as a transition point between brittle and ductile tensile behavior, and other recent work [19] used a 60\% volume fraction; Hofmann [4] used a 50\% fraction. This study selects $40 \%$ and $60 \%$ as the two levels for this factor in an effort to span an experimentally relevant and interesting region of volume fraction space. The quantity $50 \%$ serves as the center level.

\subsubsection{Crystalline Inclusion Length Scale $(L)$}

The second factor varied in this experiment is crystalline inclusion length scale $(L)$. These inclusions are modeled as circles distributed across the length and breadth of the sample. Since the inclusions need to be small enough to be roughly homogenously distributed through a sample, and the sample is only $100 \mathrm{~nm}$ in width, the two length-scale levels are both very small: this experiment uses $L=20 \mathrm{~nm}$ and $30 \mathrm{~nm}$ as the two inclusion diameter levels, with $25 \mathrm{~nm}$ as the center level. While it is again noted that these are small compared to many experiments, these crystallite length scales are on the same order as those employed in atomistic studies [41] and those experimentally observed in certain alloys [52,53]. 


\subsubsection{Yield Stress Ratio $(R)$}

The third factor varied in this experiment is the ratio of the yield stress $(R)$ between the two phases because research has shown that the ductility or brittleness of the crystalline second phase has a strong effect on strain delocalization in MGMCs [51]. This is investigated in this experiment by altering the yield stress of the crystalline phase as a fraction of the yield (failure) stress of the amorphous matrix. Hoffmann [4] uses an alloy where the crystalline yield stress is $80 \%$ of the glassy yield stress; hence, this study uses $80 \%$ as one of the levels for this factor. The second level is selected to be softer, with a crystalline yield stress $50 \%$ that of the glass yield stress. The center level is $65 \%$.

\subsection{Design of Experiment}

A body-centered design of experiment is employed, with three replicates (experimental samples with different inclusion arrangements but the same values of $V, L$, and $R$ ) at each corner and two at the center (summarized in Table 3.1). Therefore, the experiment has $2^{3}+1=9$ treatments and $3 \times 2^{3}+1 \times 2=26$ total simulations. Each experiment is analyzed for seven dependent statistics, which are described in the next section.

\subsection{Dependent (Response) Variables}

The simulated tensile tests are evaluated for both macroscopic responses (strength, ductility, and toughness) and microscopic behaviors (degree and nature of strain localization and partitioning of strain between the two MGMC phases) using seven dependent variables. These seven dependent variables are described in detail below.

\subsubsection{Maximum Stress $\left(\sigma_{\max }\right)$}

The maximum stress supported by the sample prior to failure is used as the first dependent variable. It is important since high maximum stress is what makes metallic glass structurally interesting and attractive, and the introduction of the crystalline phase typically comes at the expense of this quantity. 


\subsubsection{Tensile Strain to Failure $\left(\varepsilon_{\max }\right)$}

The sample's macroscopic strain at the failure is used as the second dependent variables in this experiment since ductility without failure is a key quality for MGMCs. Unfortunately, the model has no mechanism for failure, so a proxy variable must be selected to assess this quality. This proxy variable is selected to be the magnitude of accumulated shear strain within the metallic glass phase. Based on the notion that repeated activation of an STZ ultimately results in failure of the metallic glass matrix, when any of the amorphous elements reach a shear strain of 0.325 , the sample is considered to have failed.

\subsubsection{Strain Energy to Failure $(E)$}

The toughness of the MGMC is used as the third dependent variable, and is evaluated as the volumetric strain energy to failure. The value is obtained by integrating the area under the stress-strain curve up to the failure condition described in Section 3.3.2. This variable combines both the strength (the height of the curve) and the ductility (the length of the curve) of the sample in one convenient metric.

\subsubsection{Localization Index $(\Gamma)$}

In contrast to the previous dependent variables, the fourth dependent variable, the localization index, provides a measure of the distribution of the microscopic plasticity. This index is similar to an atomistic participation ratio, and is introduced in [44]. It is calculated:

$$
\Gamma=1-\frac{\left(\sum_{n} \gamma_{n}^{2}\right)^{2}}{N \sum_{n} \gamma_{n}^{4}}
$$

where $\gamma_{n}$ is the plastic strain associated with STZ activation in each of the $N$ elements of the sample. A value of 0 corresponds to totally uniform strain distribution, 1 corresponds to concentration of strain in an infinitely small region, and 0.5 corresponds to very homogenous flow. This metric is calculated at the failure step described in Section 3.3.2. 


\subsubsection{Two-Point Statistic Localization $\left(L_{2}\right)$}

The fifth dependent variable also evaluates the microscopic plasticity, but does so using a two-point statistical measure of strain localization. The two-point statistics of localization are obtained by first mapping the element-wise strain at the failure step to a dense, evenly-spaced twodimensional grid. At each point in the grid corresponding to metallic glass, the spectral (induced Euclidean) norm of the plastic strain tensor is computed-thus producing a rastered scalar field $P(\vec{x})$ across the sample roughly capturing the amount of accumulated plastic strain (see panel (a) of Figure 3.1 for an example of such a field). At points corresponding to crystalline inclusion, $P$ is set to zero.

The two-point statistics on $P$ are generated by autocorrelation $[54,55]$; that is, by evaluating:

$$
T(\vec{x})=\left(\frac{1}{\sqrt{n}} \mathscr{F}^{-1}\{\overline{\mathscr{F}}\{P\} \cdot \mathscr{F}\{P\}\}\right)^{(1 / 2)}
$$

where $n$ is the number of raster squares in $P, \mathscr{F}\{0\}$ is a Fast Fourier transform, overline is complex conjugation, and · is element-wise multiplication. The square root brings the units of $T$ into agreement with those of $P$; note that the $n^{-1 / 2}$ factor may require adjusting for different Fast-Fourier Transform implementations. An example of such an autocorrelation is shown as panel (b) in Figure 3.1. This map is related to the typical strain distribution around a strained point in the sample, which can be thought of as being in the center of the autocorrelation map. That is, in some sense, $T$ reduces the distributed local plastic strain fields in $P$ to a single "typical" local plastic strain field. Large, concentrated strains near the center of $T$ are indicative of high levels of localization, while less concentrated strains in $T$ suggest delocalization of strain.

The dependent variable for this two-point evaluation of localization can be reduced to a single number by averaging the strain in a square around the center of $T$, then averaging the strain everywhere else in $T$, and then taking the ratio of the two (inner/outer, see Figure 3.1). The cutoff radius between the inner and outer portions is somewhat arbitrary; experimentation suggests that $5.3 \mathrm{~nm}$ produces satisfactory results, so it is used in this study.

This metric is specifically implemented here for 2-dimensional experiments, though it could trivially be extended to 3-dimensional cases. 
(a)

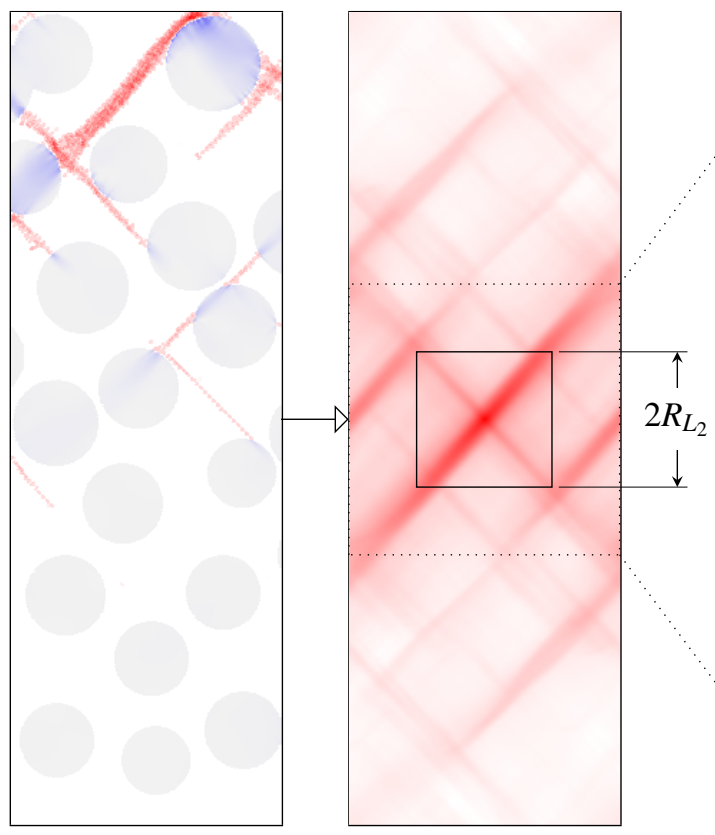

(c)

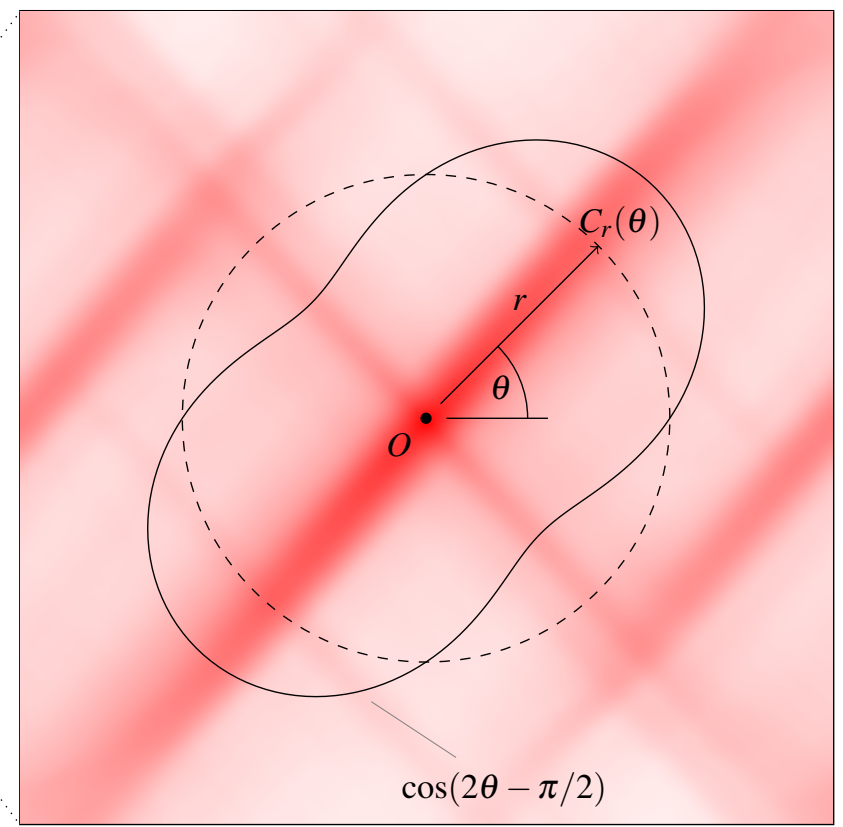

Figure 3.1: Schematic of localization analysis using two-point statistics. (a) A plot of the spectral norm of the plastic strain tensor across a sample, where white is amorphous, gray is crystalline, red is STZ plasticity, and blue is crystalline plasticity. (b) The autocorrelation of the strain field in (a), using only the amorphous portions of the mesh. The quantity $L_{2}$ is determined by computing the mean strain inside of a central box of radius $R_{L_{2}}$, and the mean strain outside of that box, and then taking the ratio of the two. (c) Schematic for computing the uniaxiality metric $U$. The function $\cos (2 \theta-\pi / 2)$ is plotted in polar coordinates with zero radius shifted to the dashed line. In this way, integral contributions along the path $C_{r}$ in the first and third quadrants are positive, and contributions in the second and fourth quadrants are negative.

\subsubsection{Uniaxiality of Strain Fields $(U)$}

The sixth dependent variable is a measure of the uniaxial or biaxial nature of typical plastic strain fields calculated by autocorrelation, since this may be correlated to strain delocalization. Path integrals of the following form can be evaluated to provide a scalar measure of the uniaxiality of the localization (i.e. does the localization tend to deflect and branch or continue in a straight line), where $C_{r}(\theta)$ is a point located at angle $\theta$ on the circle of radius $r$ centered at the origin, and 
$R$ is a cutoff radius, which in this study is set to $15.9 \mathrm{~nm}$ :

$$
U=\frac{1}{R} \int_{0}^{R}\left[\frac{\int_{0}^{2 \pi} T\left(C_{r}(\theta)\right) \cos (2 \theta-\pi / 2) d \theta}{\int_{0}^{2 \pi} T\left(C_{r}(\theta)\right) d \theta}\right] d r
$$

This integral evaluates to 0 in a case where the plastic strain field in $T$ is biaxial, to about 1 in a case where the plastic strain field is heavily biased with a positive $45^{\circ}$ slope, and about -1 in a case where the plastic strain field is heavily biased with a negative $45^{\circ}$ slope. See panel (c) of Figure 3.1.

\subsubsection{Phase Strain Average Ratio $\left(R_{\varepsilon}\right)$}

The final dependent variable is a measure of the ratio of the mean strain in the crystalline phase to the mean strain in the glassy phase as computed from the plastic strain spectral-norm map mentioned in 3.3.5. This measure indicates the degree to which the crystalline phase strains with respect to strain in the amorphous matrix.

\subsection{Summary of Variables}

To summarize, the independent variables are crystal volume fraction $(V)$, inclusion length scale $(L)$, and yield stress ratio $(R)$ between the two phases. These three independent variables permute between two levels each, with three replicates. A point at the center of all three variables is also evaluated with two replicates. The dependent variables measure strength (maximum stress, $\sigma_{\max }$ ), ductility (tensile strain to failure, $\varepsilon_{\max }$, and strain energy to failure, $E$ ), plastic localization (localization index $\Gamma$ and two-point localization $L_{2}$ ), uniaxiality of strain $(U)$, and the partitioning of strain between the two phases (phase strain average ratio $R_{\mathcal{E}}$ ). 


\section{CHAPTER 4. RESULTS}

The numerical results from the 26 experimental runs can be found in Table 4.1. This is accompanied by Figure 4.1, which presents representative plastic deformation maps for each factorial treatment, along with the stress-strain curves for all three replicates for each treatment. From these data and figures a number of trends can be observed that correspond to the various dependent variables of maximum stress, strain at failure, toughness, etc. Less intuitively, some strain distributions appear to indicate significant localization that does not always translate into expected stress drops. Fortunately, the full-factorial approach allows these relationships to be determined with statistical significance by regression fits of the dependent variables.

\subsection{Regression Analysis of Results}

To analyze the raw results presented in Table 4.1, the results are normalized by a rightpreconditioning diagonal matrix $Q$; this has the effect of dividing each measured statistic by the largest absolute value of that statistic. The preconditioned results can be found in the appendix. For simplicity, analysis in this section is done with respect to the coded independent variables (which assume values of $-1,0$, and 1 ) rather than the actual measured values.

To isolate the statistically significant trends and relationships in the data, each dependent statistic is considered in turn using regression analysis and analysis of variance (ANOVA) [50, 56, 57]. Analysis is performed using Mathematica 9.0. Only preconditioned dependent statistics are considered - this keeps the magnitudes of the statistics roughly equal and reduces risk of numerical error. An $\alpha$ level (statistical significance threshold) of 0.01 is selected for this study-that is, roughly, there is less than a $1 \%$ probability that any given regression result shown here is the result of chance alone. 


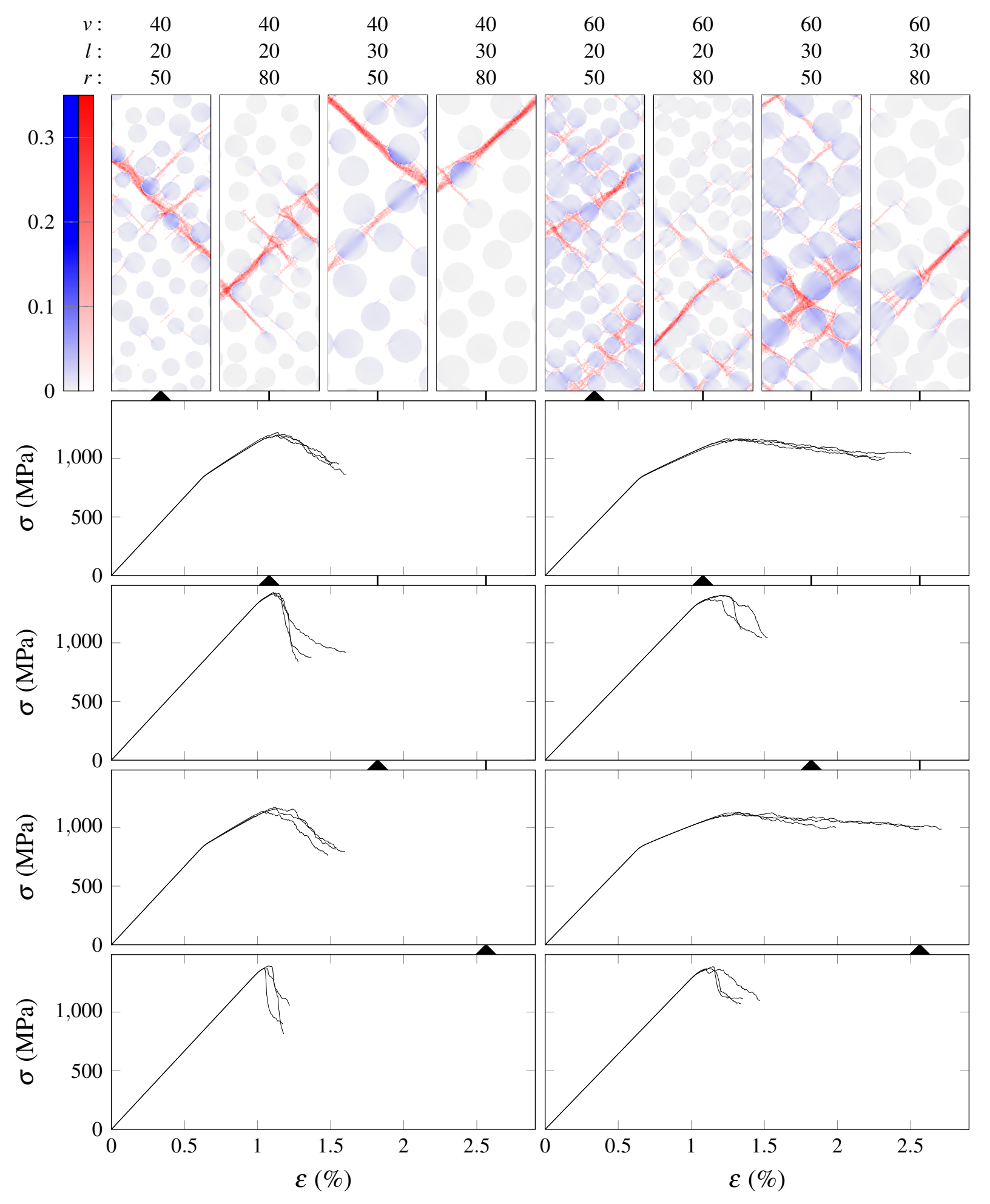

Figure 4.1: A map of the spectral norm of the plastic strain tensor is shown at failure for representatives from each of the eight full-factorial treatment groups: white is amorphous, gray is crystalline, red is STZ plasticity, and blue is crystalline plasticity. The three stress-strain curves are shown below for each treatment group. 
Table 4.1: Results from the experiment.

\begin{tabular}{|c|c|c|c|c|c|c|c|c|c|c|}
\hline \multirow[b]{2}{*}{ \# } & \multicolumn{3}{|c|}{ Ind. Vars } & \multicolumn{7}{|c|}{ Dependent Variables } \\
\hline & $v$ & $l$ & $r$ & $\sigma_{\max }$ & $\varepsilon_{\max }$ & $E$ & $\Gamma$ & $L_{2}$ & $U$ & $R_{\varepsilon}$ \\
\hline 1 & 40 & 20 & 50 & $1.22145 \times 10^{9}$ & 0.015147 & $1.21035 \times 10^{7}$ & 0.951012 & 2.27557 & 0.102155 & 1.120130 \\
\hline 2 & 40 & 20 & 50 & $1.20005 \times 10^{9}$ & 0.015570 & $1.23948 \times 10^{7}$ & 0.945286 & 2.39202 & 0.078021 & 0.950124 \\
\hline 3 & 40 & 20 & 50 & $1.20533 \times 10^{9}$ & 0.016084 & $1.29371 \times 10^{7}$ & 0.952375 & 1.97476 & 0.075796 & 0.904394 \\
\hline 4 & 40 & 20 & 80 & $1.42970 \times 10^{9}$ & 0.016041 & $1.36079 \times 10^{7}$ & 0.923209 & 2.12573 & 0.160913 & 0.311324 \\
\hline 5 & 40 & 20 & 80 & $1.42553 \times 10^{9}$ & 0.012771 & $1.02310 \times 10^{7}$ & 0.955441 & 3.43555 & 0.170527 & 0.391929 \\
\hline 6 & 40 & 20 & 80 & $1.42806 \times 10^{9}$ & 0.013684 & $1.10118 \times 10^{7}$ & 0.945380 & 3.08176 & 0.027093 & 0.299896 \\
\hline 7 & 40 & 30 & 50 & $1.17014 \times 10^{9}$ & 0.015966 & $1.23276 \times 10^{7}$ & 0.953915 & 2.35938 & 0.057653 & 0.821222 \\
\hline 8 & 40 & 30 & 50 & $1.13227 \times 10^{9}$ & 0.014811 & $1.10595 \times 10^{7}$ & 0.951899 & 2.60870 & 0.136984 & 0.854073 \\
\hline 9 & 40 & 30 & 50 & $1.16379 \times 10^{9}$ & 0.015400 & $1.19345 \times 10^{7}$ & 0.953324 & 2.31110 & 0.116054 & 1.002170 \\
\hline 10 & 40 & 30 & 80 & $1.37416 \times 10^{9}$ & 0.011802 & $8.89203 \times 10^{6}$ & 0.959090 & 3.81231 & 0.112234 & 0.262217 \\
\hline 11 & 40 & 30 & 80 & $1.39382 \times 10^{9}$ & 0.012206 & $9.45912 \times 10^{6}$ & 0.943859 & 3.45492 & 0.107956 & 0.605190 \\
\hline 12 & 40 & 30 & 80 & $1.37340 \times 10^{9}$ & 0.011725 & $8.59037 \times 10^{6}$ & 0.963572 & 6.04508 & 0.227131 & 0.219754 \\
\hline 13 & 50 & 25 & 65 & $1.29636 \times 10^{9}$ & 0.016973 & $1.45640 \times 10^{7}$ & 0.939681 & 2.01810 & 0.058977 & 0.724940 \\
\hline 14 & 50 & 25 & 65 & $1.29056 \times 10^{9}$ & 0.014752 & $1.20439 \times 10^{7}$ & 0.943955 & 2.37171 & 0.116748 & 0.590441 \\
\hline 15 & 60 & 20 & 50 & $1.16796 \times 10^{9}$ & 0.023005 & $2.00759 \times 10^{7}$ & 0.877650 & 1.47679 & 0.027822 & 0.612016 \\
\hline 16 & 60 & 20 & 50 & $1.15758 \times 10^{9}$ & 0.025050 & $2.24806 \times 10^{7}$ & 0.892223 & 1.42591 & 0.028514 & 0.635377 \\
\hline 17 & 60 & 20 & 50 & $1.16865 \times 10^{9}$ & 0.023246 & $2.06116 \times 10^{7}$ & 0.904770 & 1.37711 & 0.035653 & 0.656064 \\
\hline 18 & 60 & 20 & 80 & $1.40508 \times 10^{9}$ & 0.015221 & $1.32762 \times 10^{7}$ & 0.953116 & 2.22149 & 0.087493 & 0.407452 \\
\hline 19 & 60 & 20 & 80 & $1.40497 \times 10^{9}$ & 0.013423 & $1.10434 \times 10^{7}$ & 0.948215 & 2.93793 & 0.155172 & 0.450214 \\
\hline 20 & 60 & 20 & 80 & $1.37184 \times 10^{9}$ & 4830 & $1.24107 \times 10^{7}$ & 0.93 & 2.63275 & 0.0 & 0.253207 \\
\hline 21 & 60 & 30 & 50 & $1.12700 \times 10^{9}$ & & $2.24897 \times 10^{7}$ & & & & \\
\hline 22 & 60 & 30 & 50 & & & & & 1.6 & & 0.879816 \\
\hline 23 & 60 & 30 & 50 & & & & & 1.36 & 300 & 0.702401 \\
\hline 24 & 60 & 30 & 80 & $1.38892 \times 10^{9}$ & 0.013527 & $1.07982 \times 10^{7}$ & 0.957243 & 3.64256 & 0.075311 & 0.337341 \\
\hline 25 & 60 & 30 & 80 & $1.36923 \times 10^{9}$ & 0.014691 & $1.24378 \times 10^{7}$ & 0.928421 & 2.49704 & 0.105287 & 0.410972 \\
\hline 26 & 60 & 30 & 80 & $1.37074 \times 10^{9}$ & 0.013383 & $1.06516 \times 10^{7}$ & 0.954781 & 3.28651 & 0.169609 & 0.371147 \\
\hline
\end{tabular}
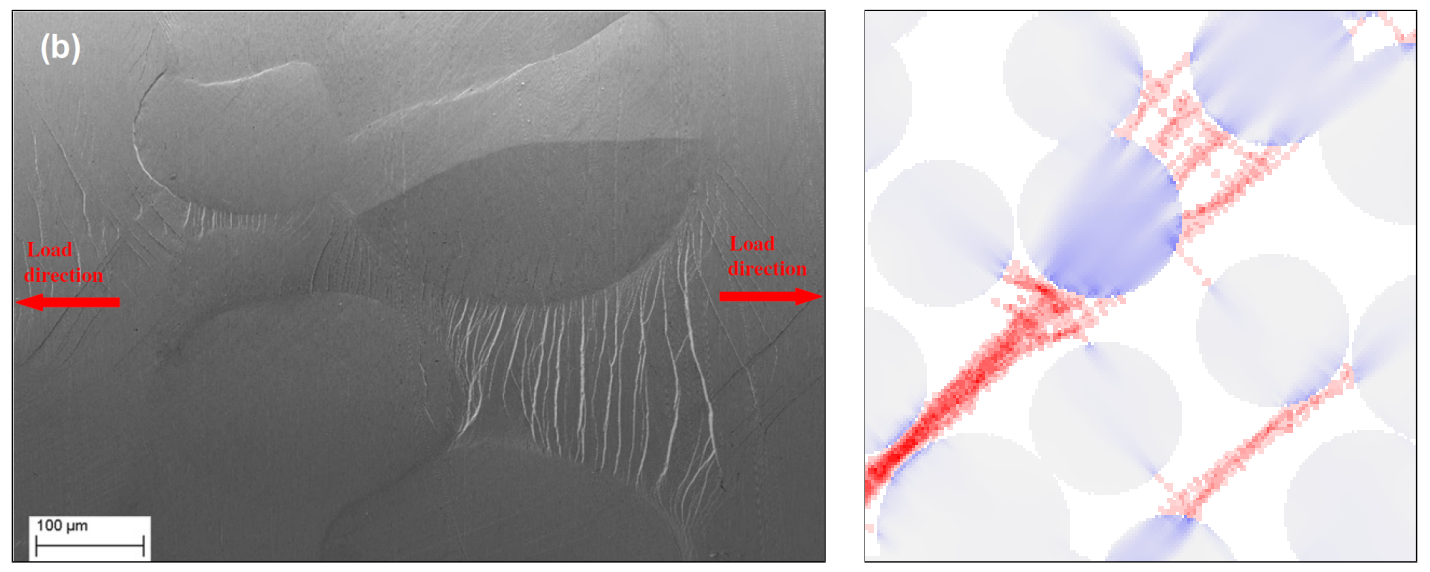

Figure 4.2: The model showed certain behaviors similar to those observed in physical samples, like the distinctive "fingers" of shear localization between phases. Micrograph from [1], used without permission. 


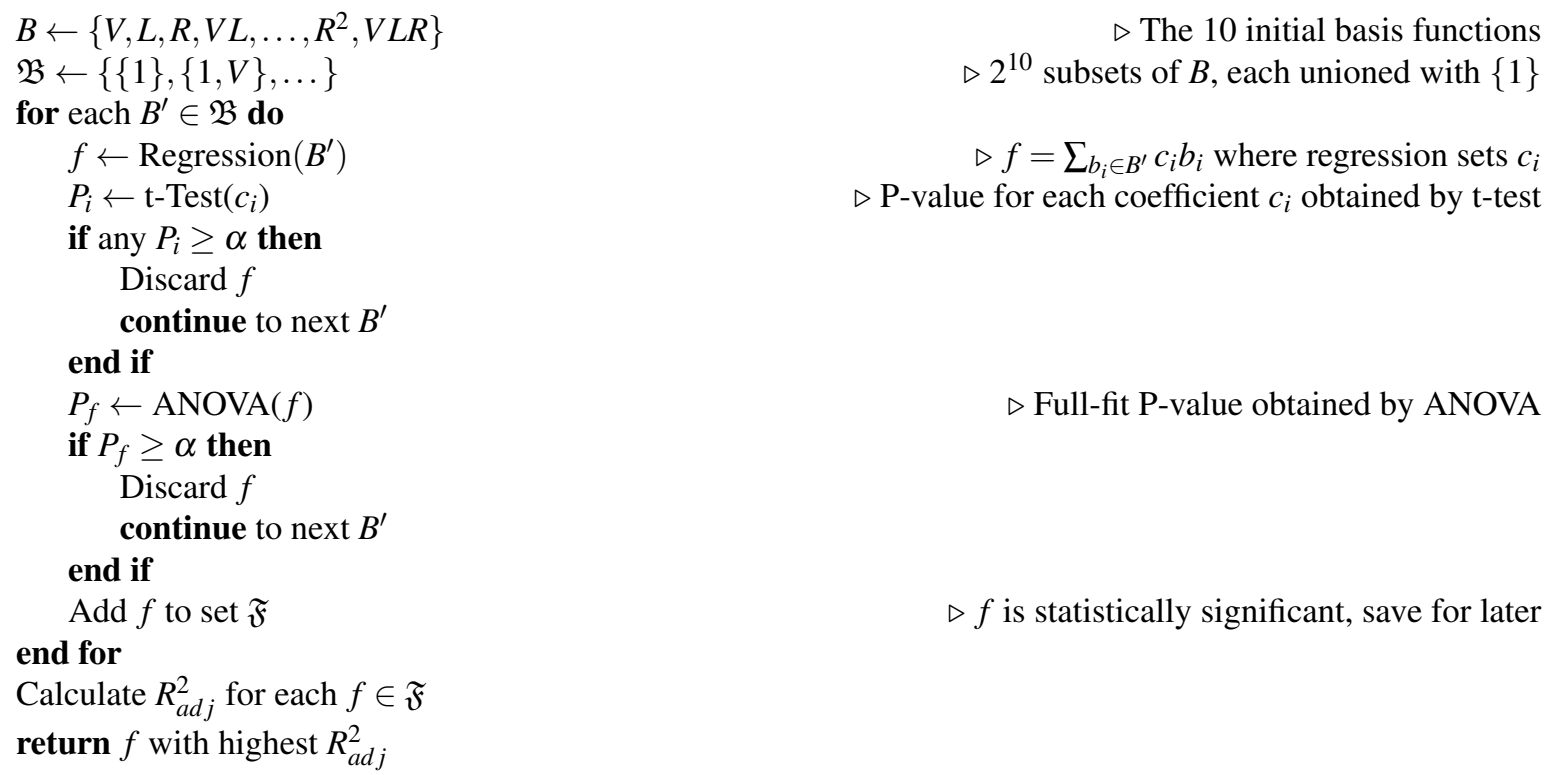

Figure 4.3: Algorithm for selecting regression model.

The following ten basis functions are initially considered to fit the data for any single dependent statistic: $\left\{V, L, R, V^{2}, L V, R V, L^{2}, L R, R^{2}, L R V\right\}$. Linear regression analysis is performed for all 1024 possible subsets (each of ten basis functions is either present or not present in a subset, for $2^{10}$ total subsets) of those basis functions. A constant basis function is also included in each regression. For each regression analysis, a t-statistic and accompanying $P$-value are computed for the coefficient to each basis function included. Models containing any regression coefficient with a $P$-value greater than $\alpha$, indicating that the coefficient did not achieve statistical significance, are discarded, ultimately leaving a small pool of potentially statistically significant regression models. Analysis of Variance (ANOVA) is performed on each of those full regression models, and any models generating an ANOVA $P$-value greater than $\alpha$ are also discarded. Out of this final pool of certainly statistically significant regression models, the model featuring the highest $R_{a d j}^{2}$ is selected for presentation in this paper. This process is outlined as an algorithm in Figure 4.3.

The full regression analyses can be found in the appendix; the regression coefficients, and significance and goodness-of-fit metrics are summarized in Table 4.2. The regression functions are visually compared to the measured data in Figure 4.4. Finally, having isolated the statistically 
Table 4.2: Summary of regressions for dependent variables. Blank entries indicate basis functions not included in regression equations. Coefficients in a column have similar scaling and can be compared to each other, though coefficients along a row have different scalings. ANOVA $\mathrm{P}$-values for the full regression fits are given, as are $R^{2}$ goodness-of-fit metrics.

\begin{tabular}{rrrrrrrr}
\hline \hline Basis & $\sigma_{\max }$ & $\varepsilon_{\max }$ & $E$ & $\Gamma$ & $L_{2}$ & $U$ & $R_{\mathcal{E}}$ \\
\hline 1 & 0.8956 & 0.6123 & 0.5704 & 0.9714 & 0.4215 & 0.3928 & 0.5325 \\
$\mathrm{~V}$ & -0.0096 & 0.0887 & 0.1076 & -0.0149 & -0.0682 & - & - \\
$\mathrm{L}$ & -0.0136 & - & - & - & - & - & - \\
$\mathrm{R}$ & 0.0806 & -0.1130 & -0.1149 & 0.0122 & 0.1136 & - & -0.2065 \\
$\mathrm{LV}$ & - & - & - & - & - & - & - \\
$\mathrm{RV}$ & - & -0.0677 & -0.0771 & 0.0137 & - & - & - \\
LR & - & - & - & - & - & - & - \\
LRV & - & - & - & - & - & - & - \\
\hline P-value & 0.0013 & 0.0014 & 0.0014 & 0.0014 & 0.0003 & - & 0.0001 \\
$R^{2}$ & 0.987 & 0.892 & 0.870 & 0.717 & 0.595 & - & 0.755 \\
\hline \hline
\end{tabular}

significant trends by regression, the implications of variation in each dependent variable can be assessed.

The regression shows that the maximum stress $\sigma_{\max }$ is heavily dependent on $R$, the crystalline phase yield stress. The maximum strain and strain energy depend about equally on $R$ and $V$, with maximum ductility at high crystalline volume fraction and low inclusion yield stress. The localization metrics $\Gamma$ and $L_{2}$ report maximum localization at low volume fraction and high inclusion yield stress. Uniaxiality $U$ does not correlate with the independent variables and partitioning of strain between phases depends only on the yield point of the second phase. 


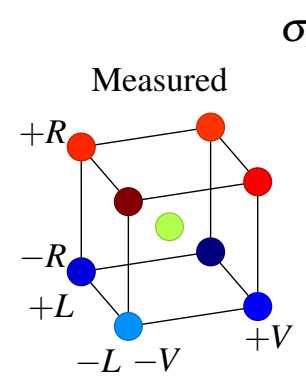

$\sigma_{\max }$
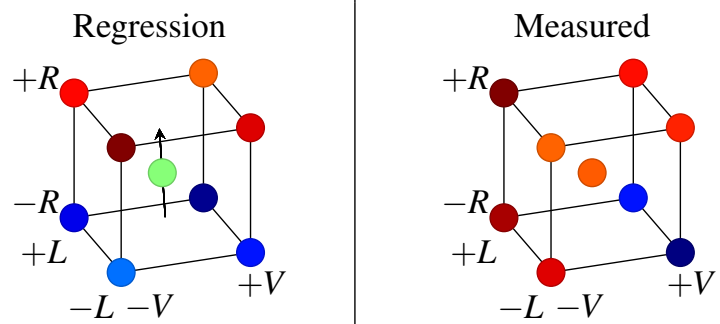

$\Gamma$
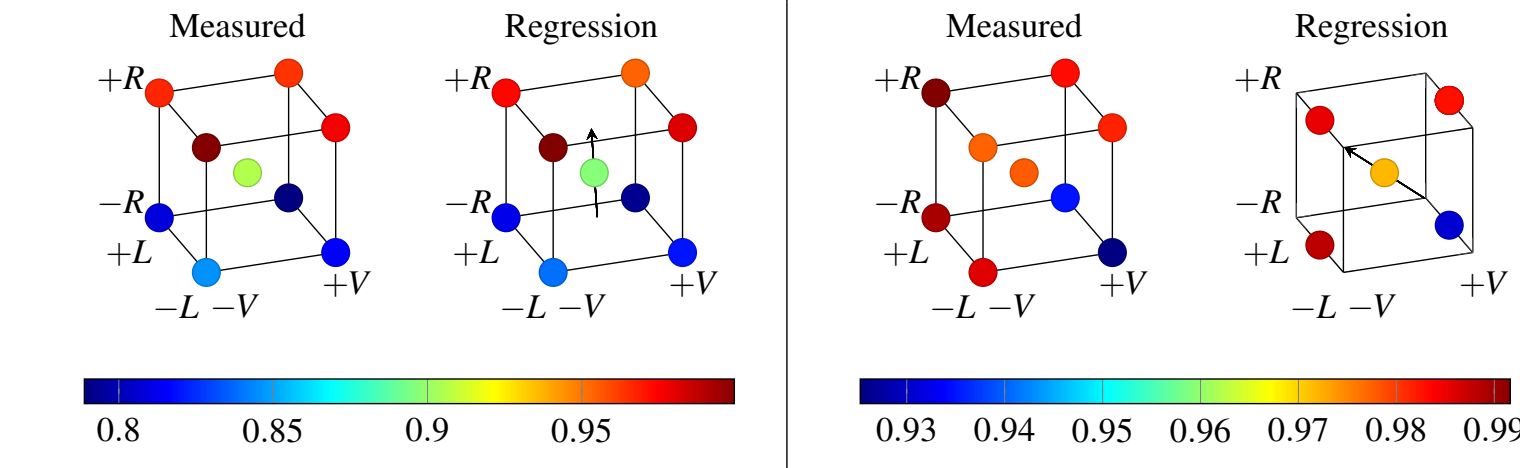

$\begin{array}{lllllll}0.93 & 0.94 & 0.95 & 0.96 & 0.97 & 0.98 & 0.99\end{array}$

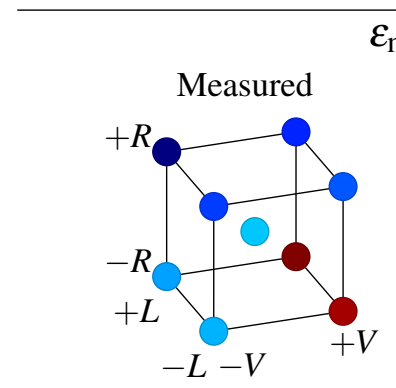

$\varepsilon_{\max }$

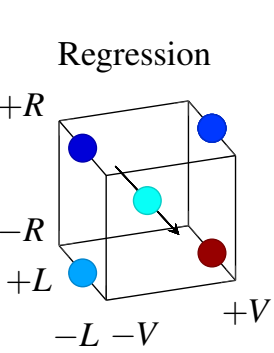

Measured $L_{2}$

2
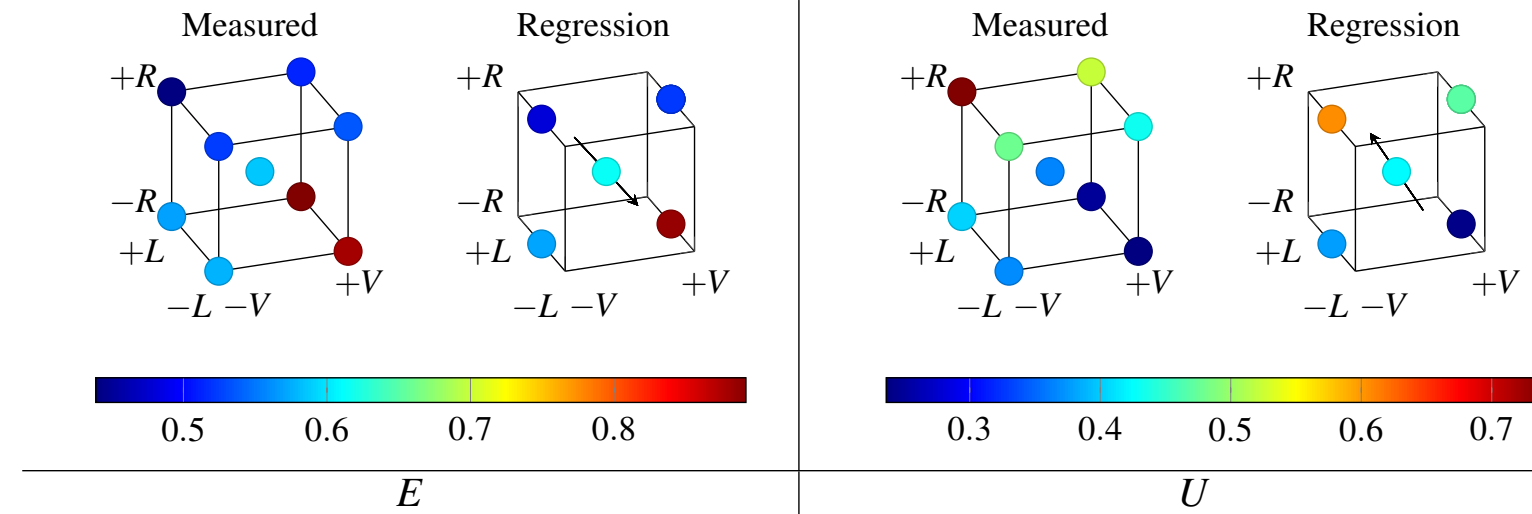

\begin{tabular}{rrrrr}
\hline 0.3 & 0.4 & 0.5 & 0.6 & 0.7 \\
\hline
\end{tabular}
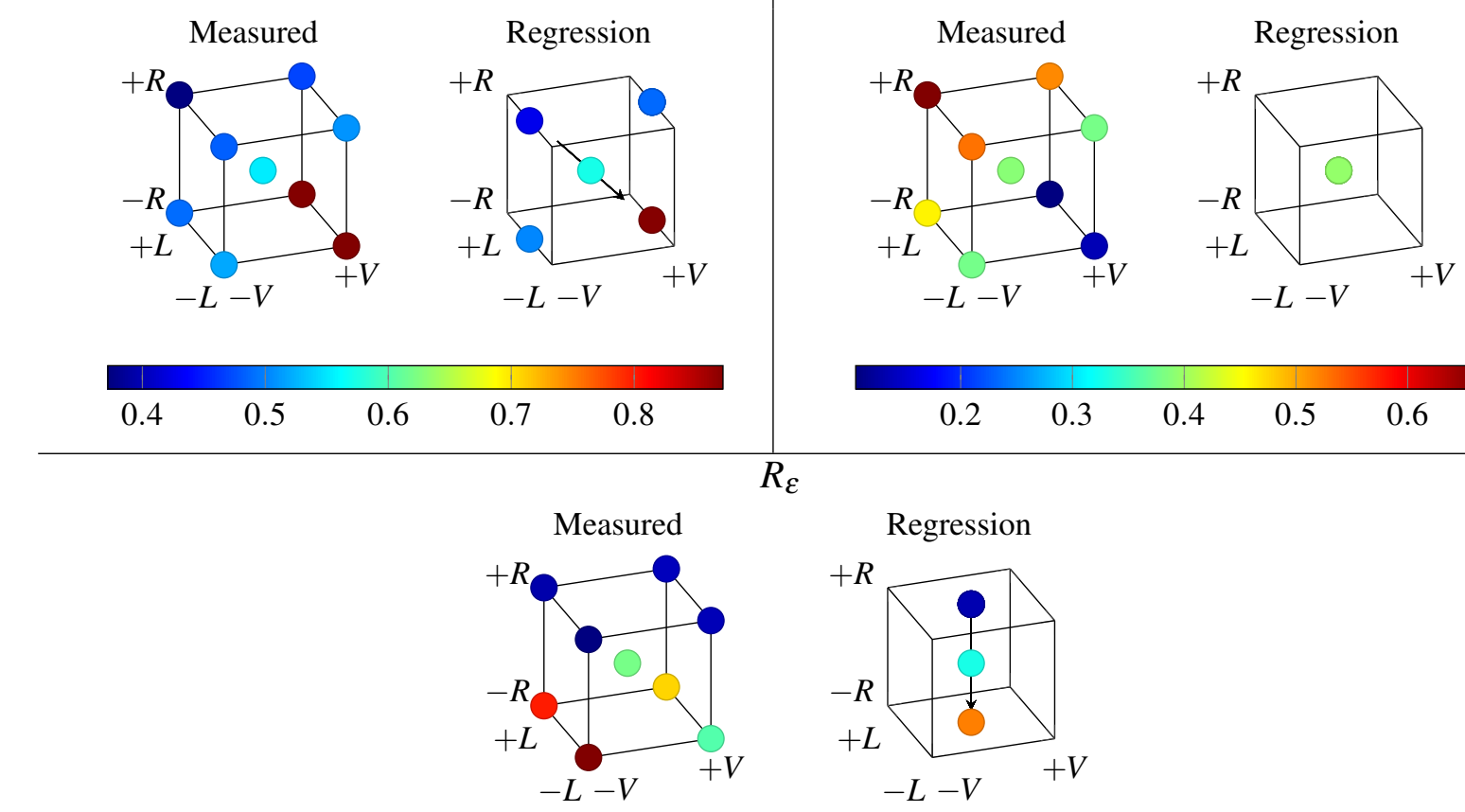

$R_{\varepsilon}$

\section{$\begin{array}{lllll}0.2 & 0.3 & 0.4 & 0.5 & 0.6\end{array}$}

Regression 


\section{CHAPTER 5. DISCUSSION}

Several distinct modes of interaction between the crystalline inclusions and shear bands in the amorphous matrix are shown and described in Figure 5.1.

The most dominant trend across all the statistics is the absence of length scale effects. Even in the one case where $L$ features in the regression equation, its coefficient (implying, roughly, its importance) is only $17 \%$ that of yield point ratio. This absence of statistically significant length scale effects may be due to the very small mean particle size and lack of contrast between $20 \mathrm{~nm}$ and $30 \mathrm{~nm}$ - in other words $20 \mathrm{~nm}$ and $30 \mathrm{~nm}$ particles are both so small, there's very little difference in effect. Regrettably, this experiment did not permit effective exploration of length scale variation, which is known to be a critical variable to MGMCs [4].

An equally dominant but opposite trend is the primacy of $R$ - the yield stress of the crystalline phase. This is probably the least-studied of the three variables, since it is (at least with intrinsic composites) the least controllable of the three in composite design. Nonetheless, the relative ductility of the second phase is apparently critical to the alloy's properties, and might prove to be a powerful design variable.

Interestingly, an $R V$ term frequently appears in regressions dominated by $R$ and $V$ terms, and is the only interaction term that proved statistically significant. The combination of these three terms can be thought of as an alias for a rule of mixtures (where $Q$ is some property):

$$
Q \propto V_{1} R_{1}+\left(1-V_{1}\right) R_{2}
$$

Least-squares regression of $\varepsilon_{\max }$ (not preconditioned) with respect to uncoded variables $v$ and $r$, using the form of Eqn (5.1) instead of the form given in Table 4.2 produces an $R^{2}$ value of 0.884 (full fit ANOVA $\mathrm{P}=0.3963$ ), indicating that about $88 \%$ of the variation in $\varepsilon_{\max }$ may be explained

by the rule of mixtures. Least-squares regression of $E$ using the form of Eqn (5.1) produces an $R^{2}$ value of 0.854 (full fit ANOVA $\mathrm{P}=0.4263$ ), and for $\Gamma$ an $R^{2}$ of 0.994 is obtained (full fit ANOVA 
(a) $\mathrm{V}=40, \mathrm{~L}=30, \mathrm{R}=80$, Rep 1

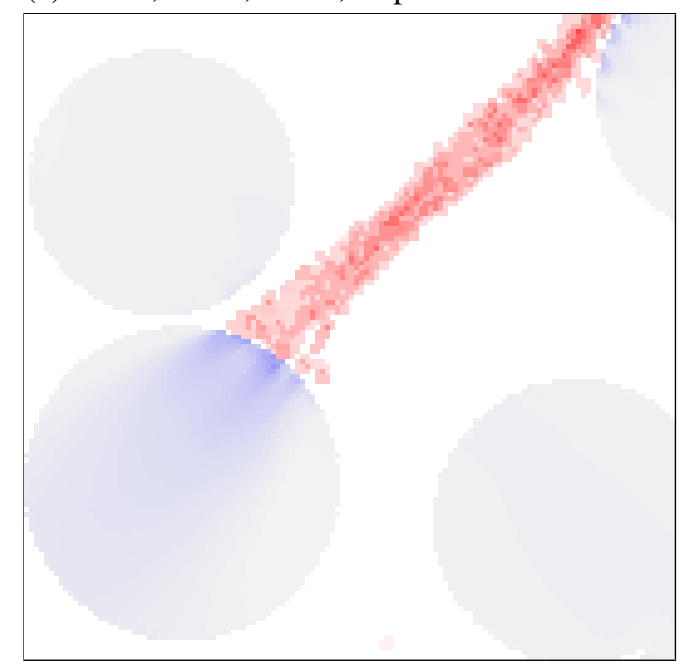

(c) $\mathrm{V}=60, \mathrm{~L}=20, \mathrm{R}=80$, Rep 2

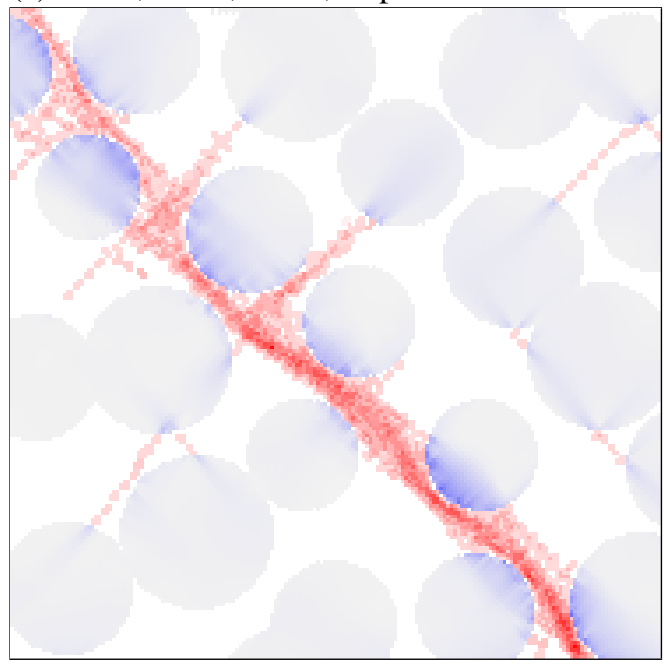

(b) $\mathrm{V}=60, \mathrm{~L}=30, \mathrm{R}=80$, Rep 3

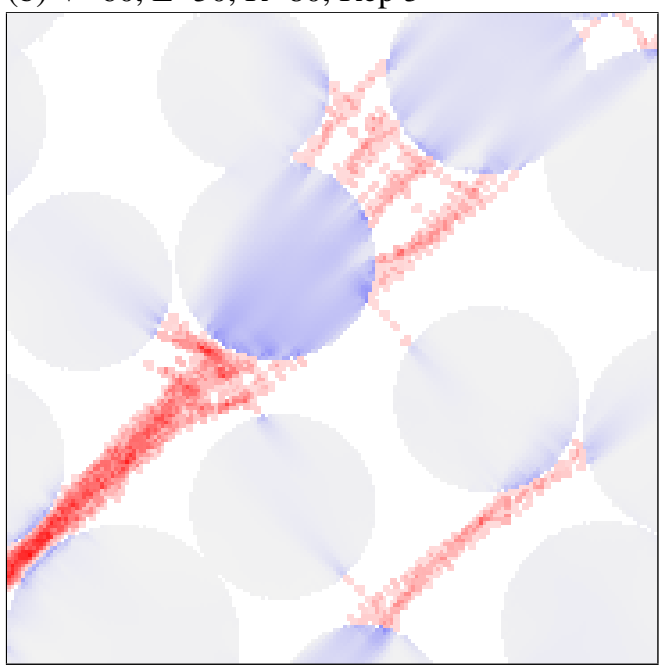

(d) $V=40, L=30, R=50$, Rep 2

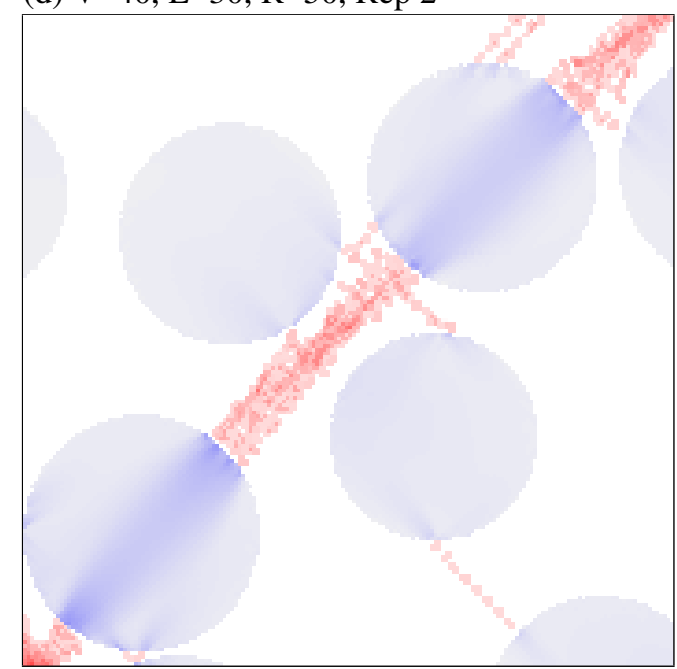

Figure 5.1: Interaction modes between shear bands and inclusions, as observed in the experiment. (a) band terminates in inclusion. (b) band fragments into smaller, less-concentrated shear bands. (c) band "pathfinds" between inclusions. (d) band penetrates inclusions.

$\mathrm{P}=0.0000$ ). It can therefore be concluded that the $\Gamma$ localization index follows a rule of mixtures with $R$ as a proxy variable; and that ductility $\left(\varepsilon_{\max }, E\right)$ requires more than a rule of mixtures explanation, even if rule of mixtures does play a role. It does seem intuitive (and experimentally consistent) that increasing the ductile volume fraction would produce more ductile composite behavior, and vice-versa.

Of course, the partitioning of strain between the two phases is secondary to the spatial concentration of - or lack thereof-strain in the amorphous matrix, since that concentration is what ultimately causes failure. The regression analysis shows that increasing volume fraction decreases 
strain localization — as would be expected — and that decreasing the yield stress of the crystalline phase also decreases strain localization. These results provide statistical evidence of the conclusion in [51] that percolation (which is largely a function of volume fraction) of the second phase is only helpful if that second phase is ductile.

The localization metric $L_{2}$ correlated strongly negatively with both $\varepsilon_{\max }$ and $E$ (Pearson product-moment correlation coefficient $r_{L_{2} X}=-0.76$ for both $X=\varepsilon_{\max }$ and $E$ ); in other words, this study shows a statistical correlation between strain delocalization and tensile ductility, as might be expected.

\subsection{Implications for Design}

This study highlights the statistical relationships between the ductility and volume fraction of the second phase, the ultimate tensile strength of the composite, and strain localization (and consequently, the failure mode) in the sample. High yield strength $(R)$ of the crystalline phase produces samples that sustain higher stress, but that exhibit a high degree of localization regardless of volume fraction. Conversely, very ductile crystalline phases produce samples with lower maximum stresses but with much less strain localization. However, this mitigation of strain localization effect is strongly dependent on volume fraction of the second phase. Designers must therefore balance the delocalizing effect of a soft second phase against the macroscopic strength of the composite, which benefits from a minimal, less ductile second phase. 


\section{CHAPTER 6. CONCLUSIONS}

The present work examines the statistical significance of microstructural factors that control plasticity in metallic glass matrix composites (MGMC). The statistical study is carried out using a model metallic glass matrix composite, which incorporates STZ-dynamics to control plasticity in the amorphous phase and a Taylor dislocation plasticity model to control plasticity in the crystalline phase.

An $\mathrm{N}$-factorial experiment is performed to investigate three microstructural variables critical to MGMC performance, namely: crystalline volume fraction, inclusion length scale, and yield strength ratio of the second phase.

Regression analysis of results indicate statistical significance of a number of results:

- Strain delocalization and the consequent ductility are facilitated by a crystalline phase with a substantially lower yield stress than that of the amorphous matrix.

- Increasing volume fraction alone is insufficient to promote strain delocalization in the case of a crystalline phase with a high relative yield stress.

- A lower yield stress for the crystalline phase implies lower maximum stresses supported by the MGMC.

These results are consistent with experimental work, but strengthen the conclusions in that the present study provides statistical evidence for these trends. The regression analysis also provides concise continuous functional forms for future MGMC constitutive equations.

\subsection{Looking Forward}

Future work on the model would include addition of free-volume to the STZ dynamics model (work already in progress [58]), and implementation of thermal gradients in the sample. 
These two additions would provide more accurate modeling of the shear-softening behavior frequently observed in bulk metallic glasses. On the crystalline side of the model, the next step is implementation of crystal plasticity to supplant the simple Taylor dislocation model in this work. More broadly, the dramatic effect of the character of the crystalline phase in this work strongly suggests the utility of experimental studies into the character of the crystalline phase across the various MGMC systems. 


\section{REFERENCES}

[1] Y. Wu, H. Wang, H. Wu, Z. Zhang, X. Hui, G. Chen, D. Ma, X. Wang, and Z. Lu, "Formation of cuzral bulk metallic glass composites with improved tensile properties," Acta Mater., vol. 59, no. 8, pp. 2928-2936, 2011. 17

[2] C. Gilbert, V. Schroeder, and R. O. Ritchie, "Mechanisms for fracture and fatigue-crack propagation in a bulk metallic glass," Metall. Mater. Trans. A, vol. 30A, pp. 1739-1753, 1999. 1

[3] M. Launey, D. C. Hofmann, J.-Y. Suh, H. Kozachkov, W. L. Johnson, and R. O. Ritchie, "Fracture toughness and crack-resistance curve behavior in metallic glass-matrix composites," Appl. Phys. Lett., vol. 94, p. 241910, 2009. 1, 2

[4] D. C. Hofmann, J.-Y. Suh, A. Wiest, G. Duan, M.-L. Lind, M. D. Demetriou, and W. L. Johnson, "Designing metallic glass matrix composites with high toughness and tensile ductility." Nature, vol. 451, pp. 1085-1089, 2008. 1, 2, 9, 10, 21

[5] C. A. Schuh and A. C. Lund, "Atomistic basis for the plastic yield criterion of metallic glass." Nature Mater., vol. 2, pp. 449-452, 2003. 1

[6] A. Argon, "Plastic deformation in metallic glasses," Acta Metall., vol. 27, pp. 47-58, 1979. 1

[7] A. Tanguy, F. Leonforte, and J.-L. Barrat, "Plastic response of a 2d lennard-jones amorphous solid: Detailed analysis of the local rearrangements at very slow strain rate," Eur. Phys. J. E, vol. 20 , no. 3, pp. 355-364, 2006. 1

[8] C. E. Maloney and A. Lemaître, "Amorphous systems in athermal, quasistatic shear," Phys. Rev. E, vol. 74, no. 1, p. $016118,2006.1$

[9] D. Rodney and C. A. Schuh, "Distribution of thermally activated plastic events in a flowing glass," Phys. Rev. Lett., vol. 102, no. 23, p. 235503, 2009. 1

[10] M. Falk and C. E. Maloney, "Simulating the mechanical response of amorphous solids using atomistic methods," Eur. Phys. J. B, vol. 75, pp. 405-413, 2010. 1

[11] D. Rodney, A. Tanguy, and D. Vandembroucq, "Modeling the mechanics of amorphous solids at different length scale and time scale," Modelling Simul. Mater. Sci. Eng., vol. 19, no. 8, p. 083001, 2011. 1, 2

[12] E. R. Homer, "Examining the initial stages of shear localization in amorphous metals," Acta Mater., p. In Press, 2013. 1, 3

[13] U. Kühn, J. Eckert, N. Mattern, and L. Schultz, "Zrnbcunial bulk metallic glass matrix composites containing dendritic bcc phase precipitates," Appl. Phys. Lett., vol. 80, pp. 2478-2480, 2002. 2 
[14] J. Cheng, G. Chen, F. Xu, Y. Du, Y. Li, and C. Liu, "Correlation of the microstructure and mechanical properties of zr-based in-situ bulk metallic glass matrix composites," Intermetallics, vol. 18, pp. 2425-2430, 2010. 2

[15] K. Qiu, A. Wang, H. Zhang, B. Ding, and Z. Hu, "Mechanical properties of tungsten fiber reinforced zralnicusi metallic glass matrix composite," Intermetallics, vol. 10, pp. 12831288, 2002. 2

[16] H. Zhang, H. Li, A. Wang, H. Fu, B. Ding, and Z. Hu, "Synthesis and characteristics of 80vol.\% tungsten (w) fibre/zr based metallic glass composite," Intermetallics, vol. 17, no. 12, pp. 1070-1077, 2009. 2, 9

[17] R. Conner, R. Dandliker, V. Scruggs, and W. L. Johnson, "Dynamic deformation behavior of tungsten-fiber/metallic-glass matrix composites," Int. J. Impact Eng., vol. 24, pp. 435-444, 2000. 2

[18] J. Qiao, H. Ye, Y. Wang, S. Pauly, H. Yang, and Z. Wang, "Distinguished work-hardening capacity of a ti-based metallic glass matrix composite upon dynamic loading," Mater. Sci. Eng. A, vol. 585, pp. 277-280, 2013. 2

[19] J. Qiao, M. Chu, L. Cheng, H. Ye, H. Yang, S. Ma, and Z. Wang, "Plastic flows of in-situ metallic glass matrix composites upon dynamic loading," Mater. Lett., vol. 119, pp. 92-95, 2014. 2,9

[20] F.-F. Wu, K. Chan, S.-T. Li, and G. Wang, "Stabilized shear banding of zrcu-based metallic glass composites under tensile loading," J. Mater. Sci., vol. 49, no. 5, pp. 2164-2170, 2014. 2

[21] J. Qiao, A. Sun, E. Huang, Y. Zhang, P. Liaw, and C. Chuang, "Tensile deformation micromechanisms for bulk metallic glass matrix composites: From work-hardening to softening," Acta Mater., vol. 59, pp. 4126-4137, 2011. 2

[22] T. Zhang, H. Ye, J. Shi, H. Yang, and J. Qiao, "Dendrite size dependence of tensile plasticity of in situ ti-based metallic glass matrix composites," J. Alloys Comp., vol. 583, pp. 593-597, 2014. 2,6

[23] J. M. Park, K. Lim, E. Park, S. Hong, K. Park, J. Eckert, and D. H. Kim, “Internal structural evolution and enhanced tensile plasticity of ti-based bulk metallic glass and composite via cold rolling," J. Alloys Comp., 2014. 2

[24] K. R. Rim, J. M. Park, W. T. Kim, and D. H. Kim, "Tensile necking and enhanced plasticity of cold rolled $\beta$-ti dendrite reinforced ti-based bulk metallic glass matrix composite," J. Alloys Comp., vol. 579, pp. 253-258, 2013. 2

[25] C. Jeon, C. Kim, S.-H. Joo, H. Kim, and S. Lee, "High tensile ductility of ti-based amorphous matrix composites modified from conventional ti6al4v titanium alloy," Acta Materialia, vol. 61, no. 8, pp. 3012-3026, 2013. 2 
[26] J. Das, S. Pauly, M. Boström, K. Durst, M. Göken, and J. Eckert, "Designing bulk metallic glass and glass matrix composites in martensitic alloys," J. Alloys Comp., vol. 483, pp. 97101, 2009. 2

[27] Y. Sun, B. Wei, Y. Wang, W. Li, T. Cheung, and C. Shek, "Plasticity-improved zr-cu-al bulk metallic glass matrix composites containing martensite phase," Appl. Phys. Lett., vol. 87, no. 5 , p. $051905,2005.2$

[28] Y. Wu, Y. Xiao, G. Chen, C. T. Liu, and Z. Lu, "Bulk metallic glass composites with transformation-mediated work-hardening and ductility." Adv. Mater., vol. 22, pp. 2770-2773, 2010. 2

[29] S. Pauly, S. Gorantla, G. Wang, U. Kühn, and J. Eckert, "Transformation-mediated ductility in cuzr-based bulk metallic glasses." Nature Mater, vol. 9, no. 6, pp. 473-477, 2010. 2

[30] J. Qiao, J. Zhang, F. Jiang, Y. Zhang, P. Liaw, Y. Ren, and G. Chen, "Development of plastic ti-based bulk-metallic-glass-matrix composites by controlling the microstructures," Mater. Sci. Eng. A, vol. 527, pp. 7752-7756, 2010. 2

[31] Y. Zhang, W. Xu, H. Tan, and Y. Li, "Microstructure control and ductility improvement of laal(cu,ni) composites by bridgman solidification," Acta Mater., vol. 53, no. 9, pp. 26072616, 2005. 2

[32] S. Pauly, J. Das, J. Bednarcik, N. Mattern, K. Kim, D. H. Kim, and J. Eckert, "Deformationinduced martensitic transformation in cu-zr-(al,ti) bulk metallic glass composites," Scripta Mater., vol. 60, pp. 431-434, 2009. 2

[33] M. Lee, Y. Li, and C. A. Schuh, "Effect of a controlled volume fraction of dendritic phases on tensile and compressive ductility in la-based metallic glass matrix composites," Acta Mater., vol. 52, pp. 4121-4131, 2004. 2, 9

[34] D. C. Hofmann, J.-Y. Suh, A. Wiest, M.-L. Lind, M. D. Demetriou, and W. L. Johnson, "Development of tough, low-density titanium-based bulk metallic glass matrix composites with tensile ductility," Proc. Natl. Acad. Sci. U. S. A., vol. 105, no. 51, pp. $20136-20140$, 2008. 2

[35] X. Fu, Y. Li, and C. A. Schuh, "Temperature, strain rate and reinforcement volume fraction dependence of plastic deformation in metallic glass matrix composites," Acta Mater., vol. 55, pp. 3059-3071, 2007. 2, 9

[36] C. Hays, C. Kim, and W. L. Johnson, "Microstructure controlled shear band pattern formation and enhanced plasticity of bulk metallic glasses containing in situ formed ductile phase dendrite dispersions," Phys. Rev. Lett., vol. 84, no. 13, pp. 2901-2904, 2000. 2

[37] J. Qiao, T. Zhang, F. Yang, P. Liaw, S. Pauly, and B. Xu, "A tensile deformation model for in-situ dendrite/metallic glass matrix composites," Sci. Rep., vol. 3, p. 2816, 2013. 2, 6

[38] R. Ott, F. Sansoz, J. Molinari, J. Almer, K. Ramesh, and T. Hufnagel, "Micromechanics of deformation of metallic-glass-matrix composites from in situ synchrotron strain measurements and finite element modeling," Acta Mater., vol. 53, pp. 1883-1893, 2005. 2 
[39] J.-C. Lee, Y.-C. Kim, J.-P. Ahn, and H. S. Kim, "Enhanced plasticity in a bulk amorphous matrix composite: Macroscopic and microscopic viewpoint studies," Acta Mater, vol. 53, pp. 129-139, 2005. 2

[40] S. Takeuchi and K. Edagawa, "Atomistic simulation and modeling of localized shear deformation in metallic glasses," Prog. Mater. Sci., vol. 56, no. 6, pp. 785-816, 2011. 2

[41] H. Zhou, S. Qu, and W. Yang, "An atomistic investigation of structural evolution in metallic glass matrix composites," Int. J. Plasticity, vol. 44, pp. 147-160, 2013. 2, 9

[42] F. Delogu, "Strain localization in metallic amorphous/amorphous composites," Intermetallics, vol. 16, no. 7, pp. 904-909, 2008. 2

[43] E. R. Homer and C. A. Schuh, "Mesoscale modeling of amorphous metals by shear transformation zone dynamics," Acta Mater., vol. 57, no. 9, pp. 2823-2833, 2009. 3, 4

[44] E. R. Homer, D. Rodney, and C. A. Schuh, "Kinetic monte carlo study of activated states and correlated shear-transformation-zone activity during the deformation of an amorphous metal," Phys. Rev. B, vol. 81, no. 6, p. 064204, 2010. 3, 11

[45] E. R. Homer and C. A. Schuh, "Three-dimensional shear transformation zone dynamics model for amorphous metals," Modelling Simul. Mater. Sci. Eng., vol. 18, no. 6, p. 065009, 2010. 3

[46] C. Packard, E. Homer, N. Al-Aqeeli, and C. Schuh, "Cyclic hardening of metallic glasses under hertzian contacts: Experiments and stz dynamics simulations," Phil. Mag., vol. 90, no. 10, pp. 1373-1390, 2010. 4, 6

[47] H. Gao, Y. Huang, W. Nix, and J. Hutchinson, "Mechanism-based strain gradient plasticity-i. theory," J. Mech. Phys. Solids, vol. 47, pp. 1239-1263, 1999. 6

[48] W. Nix and H. Gao, "Indentation size effects in crystalline materials: a law for strain gradient plasticity," J. Mech. Phys. Solids, vol. 46, no. 3, pp. 411-425, 1998. 6

[49] Y. Huang, H. Gao, W. Nix, and J. Hutchinson, "Mechanism-based strain gradient plasticity-ii . analysis," vol. 48, 2000. 6

[50] D. M. Levine, P. P. Ramsey, and R. K. Smidt, Applied Statistics for Engineers and Scientists. Prentice-Hall, 2001. 8, 15

[51] X. Fu, Y. Li, and C. A. Schuh, "Mechanical properties of metallic glass matrix composites: Effects of reinforcement character and connectivity," Scripta Mater., vol. 56, pp. 617-620, 2007. 9, 10, 23

[52] J. Das, M. Tang, K. Kim, R. Theissmann, F. Baier, W. Wang, and J. Eckert, "work-hardenable ductile bulk metallic glass," Phys. Rev. Lett., vol. 94, no. 20, p. 205501, 2005. 9

[53] K. Hajlaoui, A. Yavari, B. Doisneau, A. LeMoulec, W. Botta F., G. Vaughan, A. Greer, A. Inoue, W. Zhang, and A. Kvick, "Shear delocalization and crack blunting of a metallic glass containing nanoparticles: In situ deformation in tem analysis," Scripta Mater., vol. 54, pp. 1829-1834, 2006. 9 
[54] B. L. Adams, S. R. Kalidindi, and D. T. Fullwood, Microstructure Sensitive Design for Performance Optimization. Butterworth-Heinemann, 2013. 12

[55] D. T. Fullwood, S. R. Niezgoda, and S. R. Kalidindi, "Microstructure reconstructions from 2-point statistics using phase-recovery algorithms," Acta Mater, vol. 56, no. 5, pp. 942-948, 2008. 12

[56] J. F. Hair, R. E. Anderson, R. L. Tatham, and W. C. Black, Multivariate Data Analysis. Prentice-Hall, 1998. 15

[57] G. G. Vining, Statistical Methods for Engineers. Duxbury Press, 1998. 15

[58] L. Li, E. Homer, and C. A. Schuh, "Shear transformation zone dynamics model for metallic glasses incorporating free volume as a state variable," Acta Mater., vol. 61, no. 9, pp. 33473359, 2013. 24 


\section{APPENDIX A. CODED VARIABLES AND PRECONDITIONED RESULTS}

Table A.1: Coded independent variables and preconditioned results for regression.

\begin{tabular}{|c|c|c|c|c|c|c|c|c|c|c|}
\hline \multirow[b]{2}{*}{ \# } & \multicolumn{3}{|c|}{ Ind. Vars } & \multicolumn{7}{|c|}{ Preconditioned Dependent Variables } \\
\hline & $V$ & $L$ & $R$ & $\sigma_{\max }^{P}$ & $\varepsilon_{\max }^{P}$ & $E^{P}$ & $\Gamma^{P}$ & $L_{2}^{P}$ & $U^{P}$ & $R_{\varepsilon}^{P}$ \\
\hline 1 & -1 & -1 & -1 & 0.85434 & 0.55833 & 0.50136 & 0.98697 & 0.37643 & 0.44976 & 1.00000 \\
\hline 2 & -1 & -1 & -1 & 37 & 57393 & 0.51343 & 98102 & 0.39570 & 0.34351 & 0.84823 \\
\hline 3 & -1 & -1 & -1 & & 59287 & 0.53589 & 0.98838 & 0.32667 & 0.33371 & 0.80740 \\
\hline 4 & -1 & -1 & +1 & 1.00000 & 59129 & 0.56367 & 0.95811 & 0.35165 & 0.70846 & 0.27794 \\
\hline 5 & -1 & -1 & +1 & 08 & 47075 & 0.42380 & 0.99156 & 0.56832 & 0.75079 & 0.34990 \\
\hline 6 & -1 & -1 & +1 & 0.99885 & 50441 & 0.45614 & 0.98112 & 0.50980 & 0.11928 & 0.26773 \\
\hline 7 & -1 & +1 & -1 & & 52 & 0.51064 & 8998 & 0.39030 & 0.25383 & 0.73315 \\
\hline 8 & -1 & +1 & -1 & & 95 & 0.45811 & 8789 & 0.43154 & 0.60311 & 0.76248 \\
\hline 9 & -1 & +1 & -1 & & & 0.49436 & & 0.38231 & 0.5 & 0.89470 \\
\hline 10 & -1 & +1 & +1 & & & 0.36833 & & 0.63065 & & 0.2 \\
\hline 11 & -1 & +1 & +1 & & & 0.39182 & 54 & 0.57153 & 0.4 & 0.54029 \\
\hline 12 & -1 & +1 & +1 & & & 0.35 & 1.00000 & 1.00000 & 1.00000 & 0.19619 \\
\hline 13 & 0 & 0 & 0 & & & & & 0.33384 & & 0.64719 \\
\hline 14 & 0 & 0 & 0 & & & 39 & 64 & 0.39234 & & 0.52712 \\
\hline 15 & +1 & -1 & -1 & & & 50 & & 30 & 49 & 0.54638 \\
\hline 16 & +1 & -1 & -1 & & & 21 & 95 & 0.2 & 54 & 0.56724 \\
\hline 17 & +1 & -1 & -1 & & & & & 0.2 & 97 & 0.58571 \\
\hline 18 & +1 & -1 & +1 & & & 0.54 & 15 & 0.36749 & 0.38 & 0.36376 \\
\hline 19 & +1 & -1 & +1 & & & & .06 & 0.48600 & 0.6 & 0.40193 \\
\hline 20 & +1 & -1 & +1 & & 65 & 0.51408 & 021 & 0.43552 & 0.06230 & 0.22605 \\
\hline 21 & +1 & +1 & -1 & & 0.94298 & 0.93158 & 877 & 0.25133 & 0.02620 & 0.65570 \\
\hline 22 & +1 & +1 & -1 & & 83 & 0.68138 & 7709 & 0.26573 & 0.25555 & 0.78546 \\
\hline 23 & +1 & +1 & -1 & & 1.00000 & 1.00000 & 0.90835 & 0.22559 & 0.03654 & 0.62707 \\
\hline 24 & +1 & +1 & +1 & & 0.49862 & 0.44729 & 9343 & 0.60257 & 0.33158 & 0.30116 \\
\hline 25 & +1 & +1 & +1 & 0.95770 & 0.54152 & 0.51521 & 0.96352 & 0.41307 & 0.46355 & 0.36690 \\
\hline 26 & +1 & +1 & +1 & 0.95876 & 0.49331 & 0.44122 & 0.99088 & 0.54367 & 0.74675 & 0.33134 \\
\hline \multicolumn{4}{|c|}{ Preconditioners: } & $6.995 \times 10^{-10}$ & 36.86 & $4.142 \times 10^{-8}$ & 1.038 & 0.1654 & 4.403 & 0.8928 \\
\hline
\end{tabular}




\section{APPENDIX B. REGRESSION DETAILS}

\section{B.1 Maximum Stress $\left(\sigma_{\max }\right)$}

The regression model for $\sigma_{\max }^{P}$ uses the set $\{1, V, L, R\}$ as its basis functions. Their leastsquares coefficients, t-statistics, and P-values are shown in Table B.1, along with ANOVA results for the full model. All P-values are less than $\alpha$, signifying that all coefficients and the regression itself are statistically significant.

Table B.1: Regression analysis for $\sigma_{\max }^{P}$ :

$$
R^{2}=0.987, R_{a d j}^{2}=0.985, s=0.0098 \text {. }
$$

\begin{tabular}{|c|c|c|c|c|c|c|}
\hline \multicolumn{7}{|c|}{ Regression Coefficients } \\
\hline Basis & \multirow{2}{*}{\multicolumn{2}{|c|}{$\begin{array}{l}\text { Estimated } \\
\text { Coefficient }\end{array}$}} & \multicolumn{3}{|c|}{ Standard } & \multirow[b]{2}{*}{ P-Value } \\
\hline Function & & & Err & & t-Statistic & \\
\hline 1 & \multicolumn{2}{|c|}{0.895642} & \multicolumn{2}{|c|}{0.00192442} & 465.41 & 0.0000 \\
\hline V & \multicolumn{2}{|c|}{-0.00959846} & \multicolumn{2}{|c|}{0.002003} & -4.79205 & 0.0001 \\
\hline $\mathrm{L}$ & \multicolumn{2}{|c|}{-0.0135911} & \multicolumn{2}{|c|}{0.002003} & -6.7854 & 0.0000 \\
\hline $\mathrm{R}$ & \multicolumn{2}{|c|}{0.0805778} & \multicolumn{2}{|c|}{0.002003} & 40.2286 & 0.0000 \\
\hline \multicolumn{7}{|c|}{ Analysis of Variance } \\
\hline Source & DF & SS & & MS & $\mathrm{F}$ & $\mathrm{P}$ \\
\hline Regression & 3 & 542. & & 180.949 & 7.39599 & 0.0013 \\
\hline Error & 22 & 538. & & 24.4658 & & \\
\hline Total & 25 & 0.16 & & & & \\
\hline
\end{tabular}




\section{B.2 Tensile Strain to Failure $\left(\varepsilon_{\max }\right)$}

The regression model for $\varepsilon_{\max }^{P}$ uses the set $\{1, V, R, R V\}$ as its basis functions. Their leastsquares coefficients, t-statistics, and P-values are shown in Table B.2, along with ANOVA results for the full model. All P-values are less than $\alpha$, signifying that all coefficients and the regression itself are statistically significant.

Table B.2: Regression analysis for $\varepsilon_{\max }^{P}$ :

$$
R^{2}=0.892, R_{a d j}^{2}=0.878, s=0.0575 \text {. }
$$

\begin{tabular}{|c|c|c|c|c|c|c|c|}
\hline \multicolumn{8}{|c|}{ Regression Coefficients } \\
\hline Basis & \multirow{2}{*}{\multicolumn{2}{|c|}{$\begin{array}{l}\text { Estimated } \\
\text { Coefficient }\end{array}$}} & \multicolumn{4}{|c|}{ Standard } & \multirow[b]{2}{*}{ P-Value } \\
\hline Function & & & Error & & $\mathrm{t}-\mathrm{St}$ & atistic & \\
\hline 1 & \multicolumn{2}{|c|}{0.612317} & \multicolumn{2}{|c|}{0.0112864} & \multicolumn{2}{|c|}{54.2528} & 0.0000 \\
\hline V & \multicolumn{2}{|c|}{0.0887135} & 0.011 & 472 & 7.5 & 187 & 0.0000 \\
\hline $\mathrm{R}$ & \multicolumn{2}{|c|}{-0.112989} & 0.011 & 472 & -9.6 & 184 & 0.0000 \\
\hline R V & \multicolumn{2}{|c|}{-0.0676843} & 0.011 & 472 & 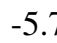 & 6173 & 0.0000 \\
\hline \multicolumn{8}{|c|}{ Analysis of Variance } \\
\hline Source & DF & SS & & MS & & $\mathrm{F}$ & $\mathrm{P}$ \\
\hline Regression & 3 & 1.4047 & $\times 10^{6}$ & 468 & & 7.33403 & 0.0014 \\
\hline Error & 22 & 1.4045 & $\times 10^{6}$ & & & & \\
\hline Total & 25 & 0.6780 & & & & & \\
\hline
\end{tabular}




\section{B.3 Strain Energy to Failure $(E)$}

The regression model for $E^{P}$ uses the set $\{1, V, R, R V\}$ as its basis functions. Their leastsquares coefficients, t-statistics, and P-values are shown in Table B.3, along with ANOVA results for the full model. All P-values are less than $\alpha$, signifying that all coefficients and the regression itself are statistically significant.

Table B.3: Regression analysis for $E^{P}$ :

$$
R^{2}=0.870, R_{a d j}^{2}=0.852, s=0.0709 \text {. }
$$

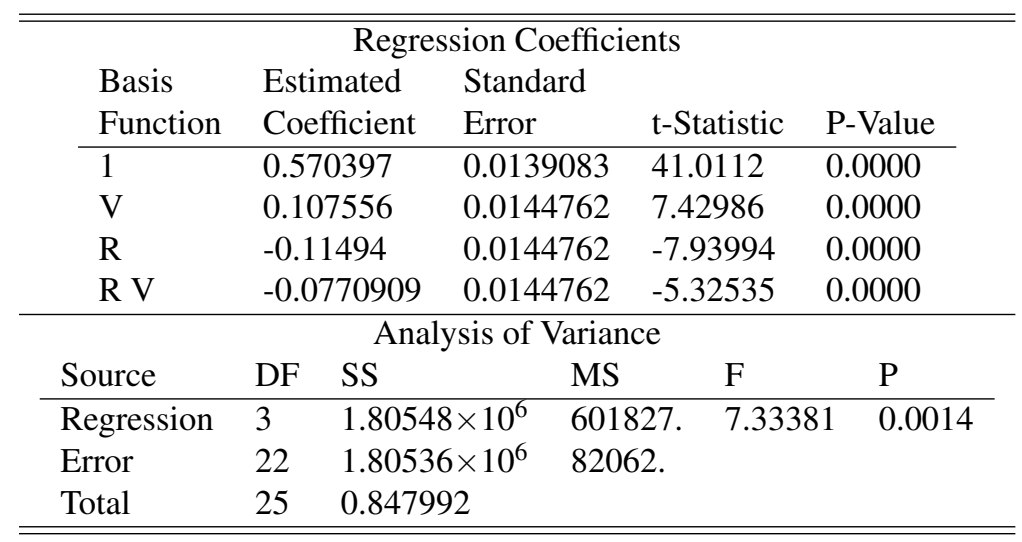




\section{B.4 Localization Index $(\Gamma)$}

The regression model for $\Gamma^{P}$ uses the set $\{1, V, R, R V\}$ as its basis functions. Their leastsquares coefficients, t-statistics, and P-values are shown in Table B.4, along with ANOVA results for the full model. All P-values are less than $\alpha$, signifying that all coefficients and the regression itself are statistically significant.

Table B.4: Regression analysis for $\Gamma^{P}$ :

$$
R^{2}=0.717, R_{a d j}^{2}=0.678, s=0.0155 \text {. }
$$

\begin{tabular}{|c|c|c|c|c|c|c|}
\hline \multicolumn{7}{|c|}{ Regression Coefficients } \\
\hline \multirow{2}{*}{$\begin{array}{l}\text { Basis } \\
\text { Function }\end{array}$} & \multirow{2}{*}{\multicolumn{2}{|c|}{$\begin{array}{l}\text { Estimated } \\
\text { Coefficient }\end{array}$}} & \multicolumn{3}{|c|}{ Standard } & \multirow[b]{2}{*}{ P-Value } \\
\hline & & & \multicolumn{2}{|c|}{ Error } & t-Statistic & \\
\hline 1 & \multicolumn{2}{|c|}{0.971359} & 0.00 & 04519 & 318.982 & 0.0000 \\
\hline $\mathrm{V}$ & -0.014 & 9193 & 0.00 & 16953 & -4.70709 & 0.0001 \\
\hline $\mathrm{R}$ & 0.0122 & 237 & 0.00 & 16953 & 3.85661 & 0.0009 \\
\hline R V & 0.0137 & 163 & 0.00 & 16953 & 4.32756 & 0.0003 \\
\hline \multicolumn{7}{|c|}{ Analysis of Variance } \\
\hline Source & DF & SS & & MS & $\mathrm{F}$ & $\mathrm{P}$ \\
\hline Regression & 3 & 563 & & 18785.7 & 7.33349 & 0.0014 \\
\hline Error & 22 & 563 & & 2561.64 & & \\
\hline Total & 25 & 0.01 & 7476 & & & \\
\hline
\end{tabular}




\section{B.5 Two-Point Statistic Localization $\left(L_{2}\right)$}

The regression model for $L_{2}^{P}$ uses the set $\{1, V, R\}$ as its basis functions. Their least-squares coefficients, t-statistics, and P-values are shown in Table B.5, along with ANOVA results for the full model. All P-values are less than $\alpha$, signifying that all coefficients and the regression itself are statistically significant.

Table B.5: Regression analysis for $L_{2}^{P}$ :

$$
R^{2}=0.595, R_{a d j}^{2}=0.560, s=0.1117 \text {. }
$$

\begin{tabular}{|c|c|c|c|c|c|c|}
\hline \multicolumn{7}{|c|}{ Regression Coefficients } \\
\hline \multirow{2}{*}{$\begin{array}{l}\text { Basis } \\
\text { Function }\end{array}$} & \multirow{2}{*}{\multicolumn{2}{|c|}{$\begin{array}{l}\text { Estimated } \\
\text { Coefficient }\end{array}$}} & \multicolumn{2}{|c|}{ Standard } & \multirow[b]{2}{*}{ t-Statistic } & \multirow[b]{2}{*}{ P-Value } \\
\hline & & & Err & & & \\
\hline 1 & 0.421 & & 0.0 & 18966 & 19.2514 & 0.0000 \\
\hline V & -0.068 & 1646 & 0.0 & 27907 & -2.9909 & 0.0065 \\
\hline $\mathrm{R}$ & 0.113 & & & 27907 & 4.985 & 0.0000 \\
\hline \multicolumn{7}{|c|}{ Analysis of Variance } \\
\hline Source & DF & SS & & MS & $\mathrm{F}$ & $\mathrm{P}$ \\
\hline Regression & 2 & 491. & & 245.99 & 11.7585 & 0.0003 \\
\hline Error & 23 & 481. & & 20.920 & & \\
\hline Total & 25 & 0.70 & 012 & & & \\
\hline
\end{tabular}




\section{B.6 Uniaxiality $(U)$}

None of the potential regression models for $U$ achieved $P<\alpha$ for all parameters - so the regression equation is simply $U \approx 0.392784$, with $s=0.2542$.

\section{B.7 Phase Strain Average Ratio $\left(R_{\varepsilon}\right)$}

The regression model for $R_{\mathcal{E}}^{P}$ uses the set $\{1, R\}$ as its basis functions. Their least-squares coefficients, $\mathrm{t}$-statistics, and P-values are shown in Table B.6, along with ANOVA results for the full model. All P-values are less than $\alpha$, signifying that all coefficients and the regression itself are statistically significant.

Table B.6: Regression analysis for $R_{\varepsilon}^{P}$ :

$$
R^{2}=0.755, R_{a d j}^{2}=0.745, s=0.1175 \text {. }
$$

\begin{tabular}{llllll}
\hline \hline \multicolumn{5}{c}{ Regression Coefficients } \\
Basis & Estimated & Standard \\
Function & Coefficient & Error & t-Statistic & P-Value \\
\hline 1 & 0.532504 & 0.0230476 & 23.1046 & 0.0000 \\
$\mathrm{R}$ & -0.20651 & 0.0239887 & -8.60864 & 0.0000 \\
\hline \multicolumn{7}{c}{ Analysis of Variance } \\
Source & DF & SS & MS & F & P \\
\hline Regression & 1 & 4914.98 & 4914.98 & 24.1442 & 0.0001 \\
Error & 24 & 4885.63 & 203.568 & & \\
Total & 25 & 1.35497 & & & \\
\hline \hline
\end{tabular}


APPENDIX C. STRAIN FIELDS AND TWO-POINT CORRELATIONS FOR ALL RUNS

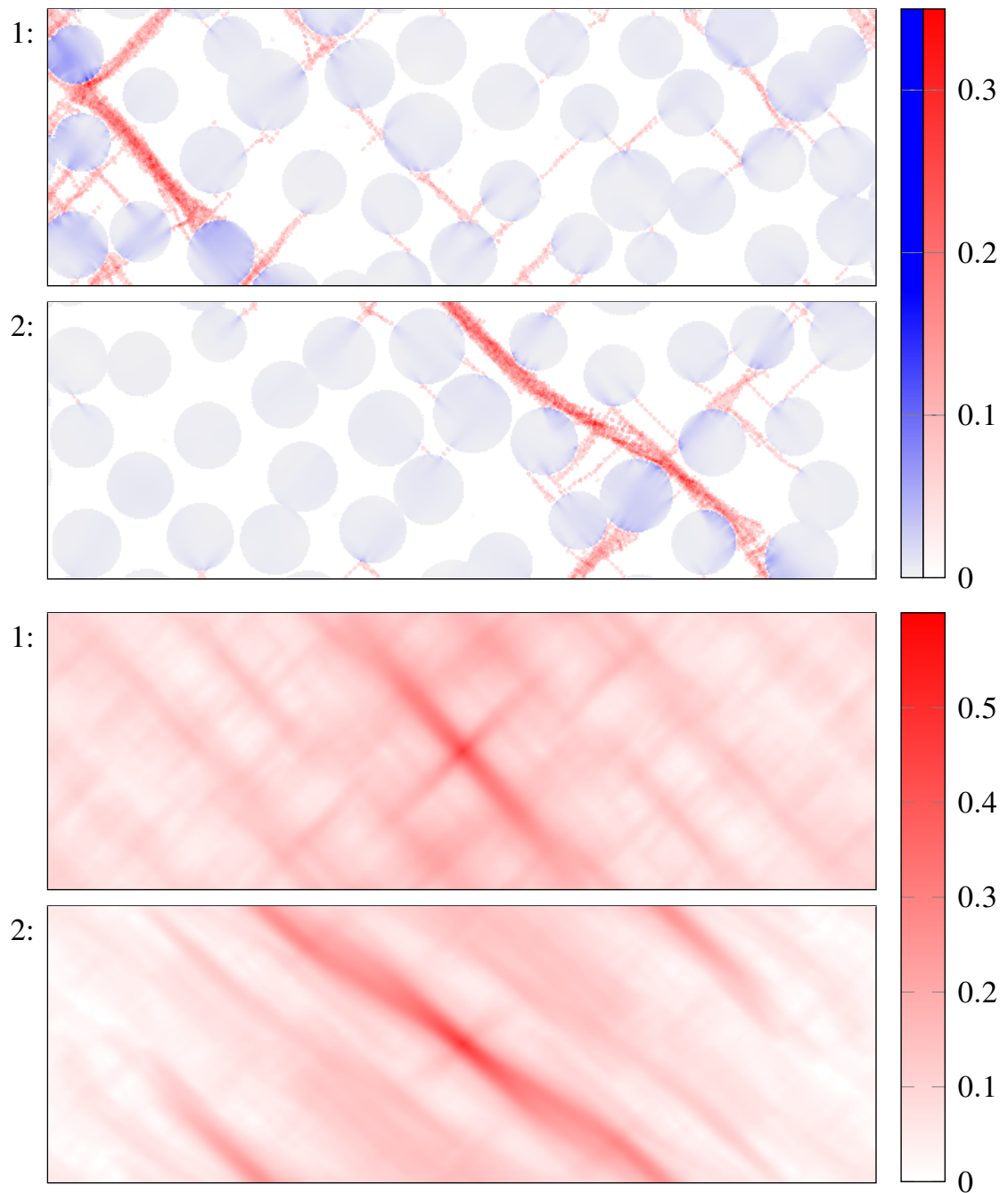

Figure C.1: Strain and two-point localization plot for $v=50, l=25, r=65$. 


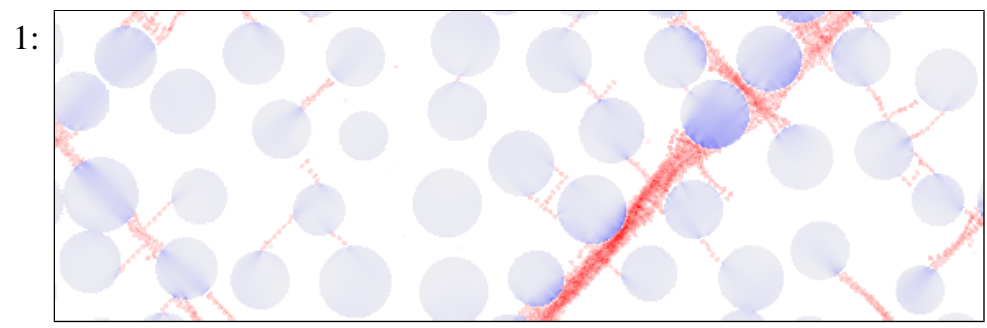

2:

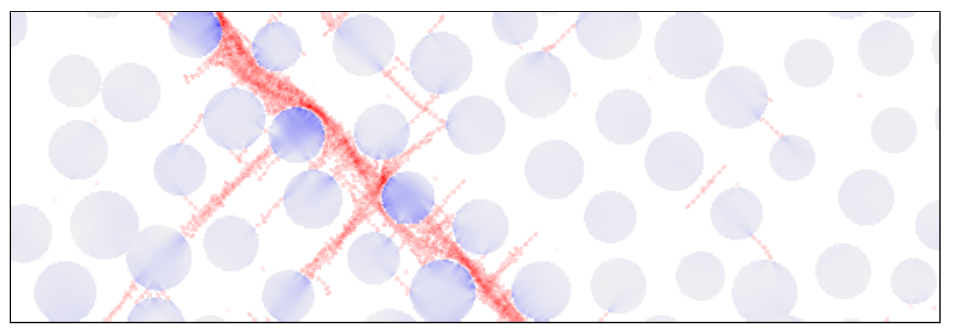

3:
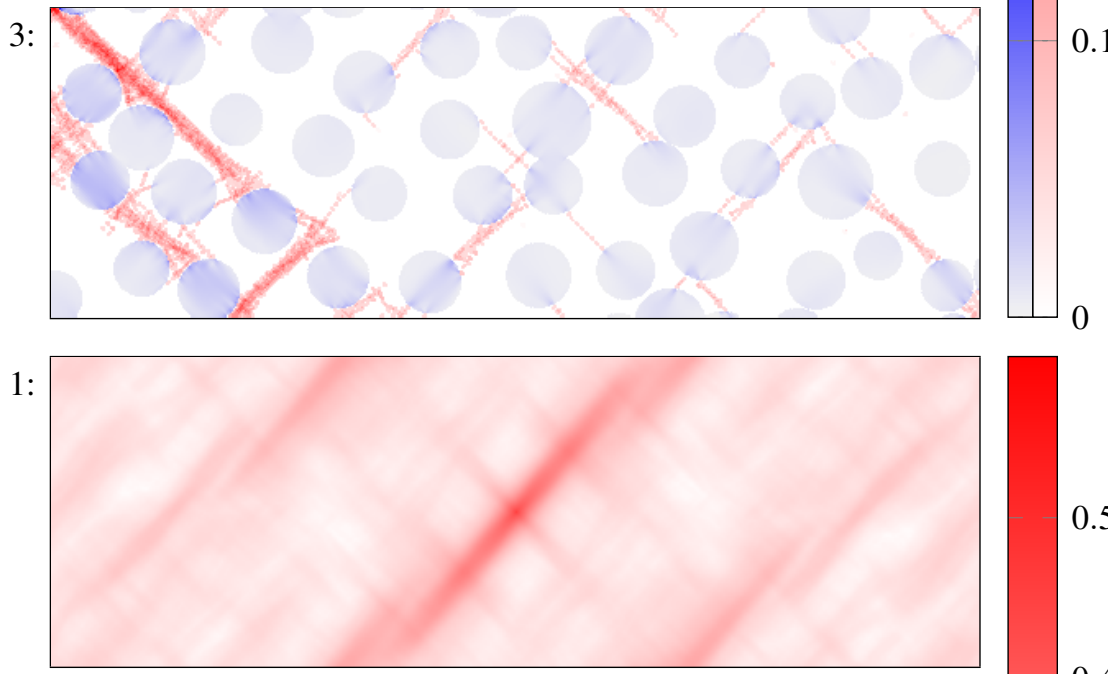

2:

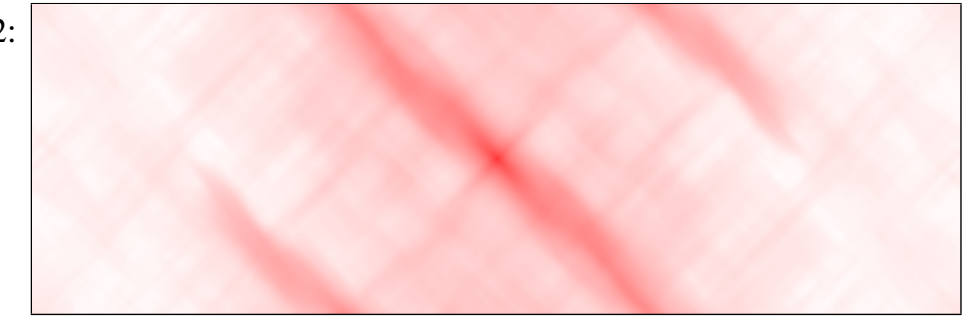

0.4

3:

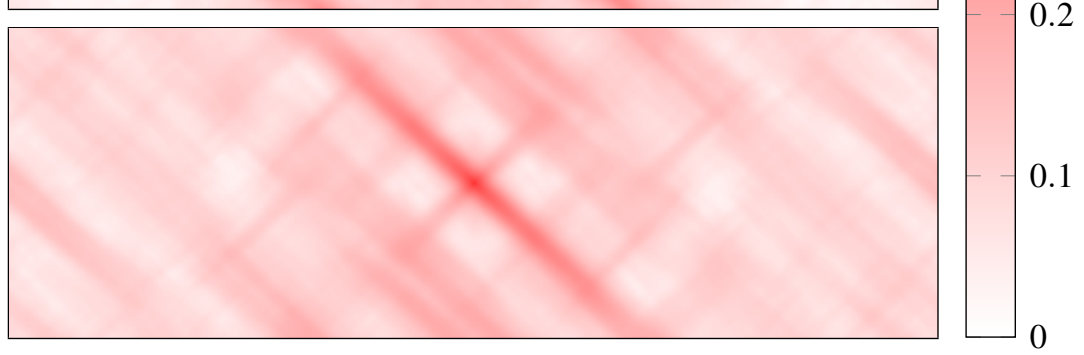

Figure C.2: Strain and two-point localization plot for $v=40, l=20, r=50$. 
$1:$

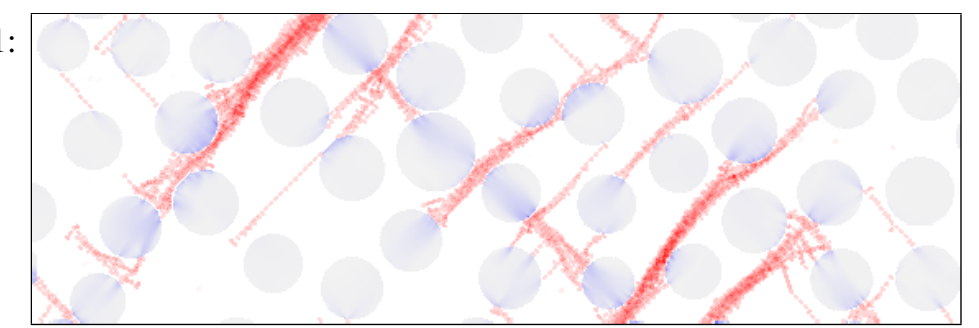

2:

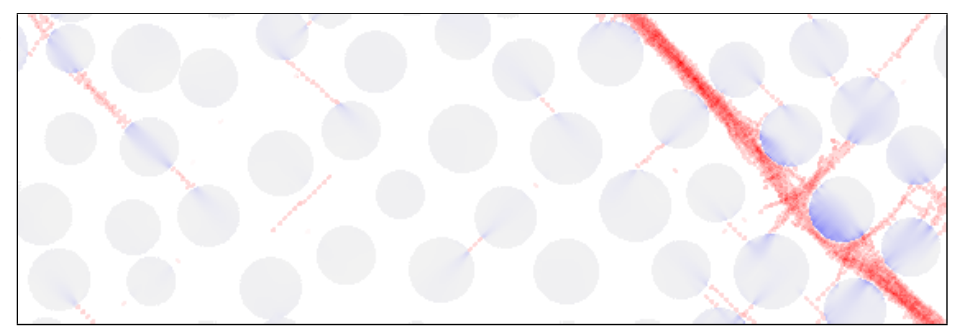

3:
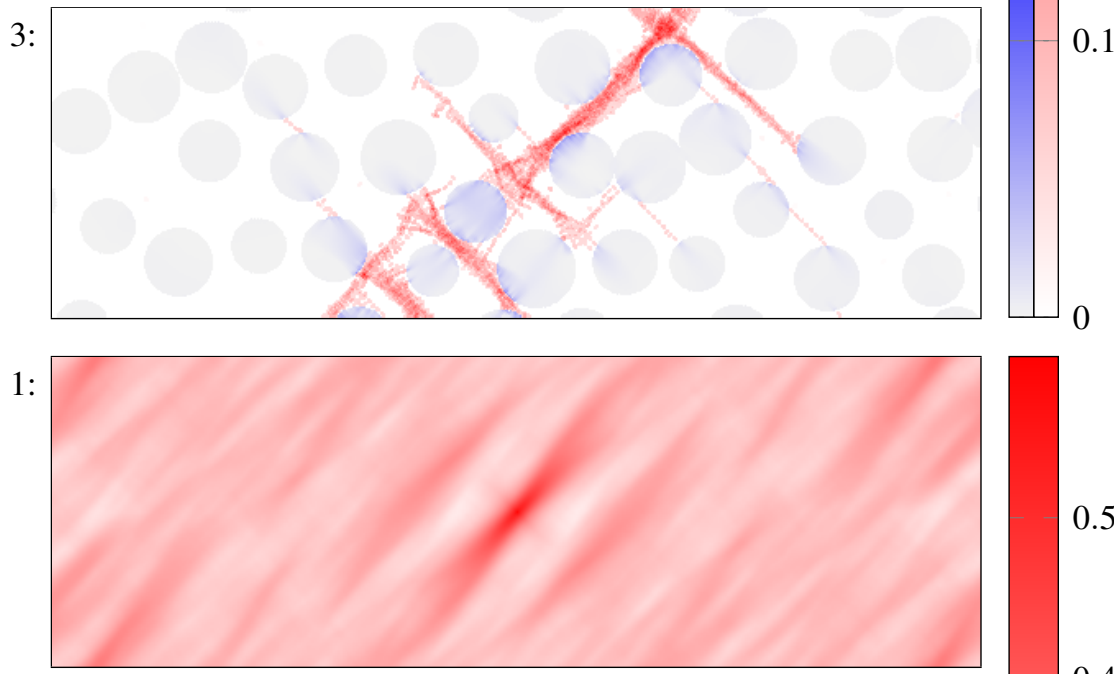

2:

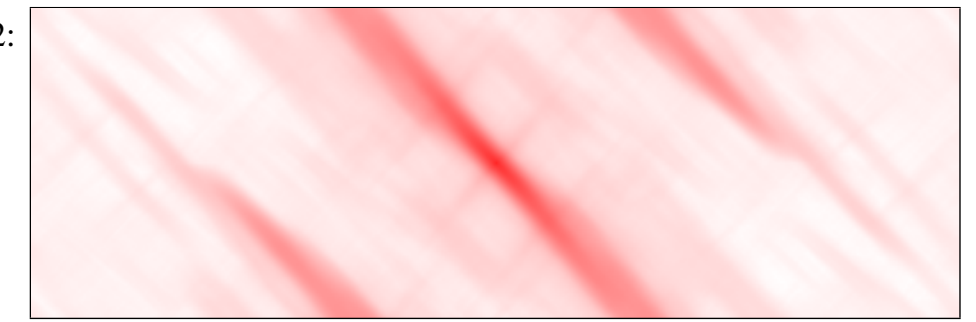

0.4

3:

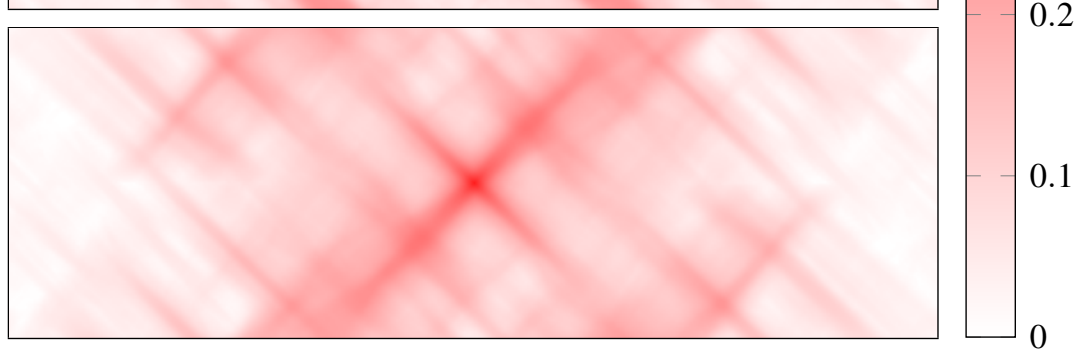

Figure C.3: Strain and two-point localization plot for $v=40, l=20, r=80$. 


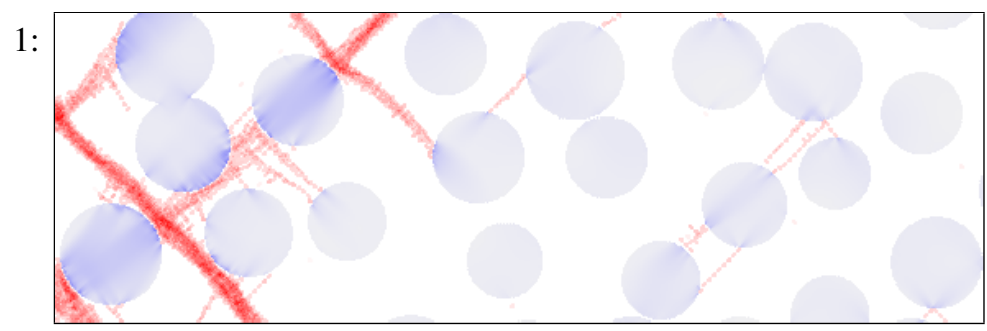

2:

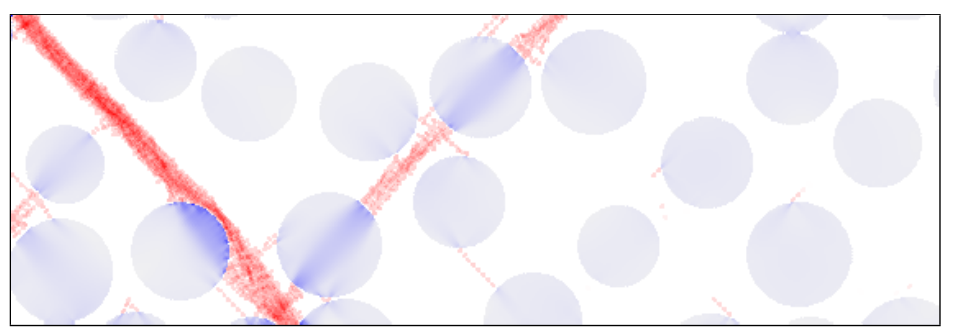

3:
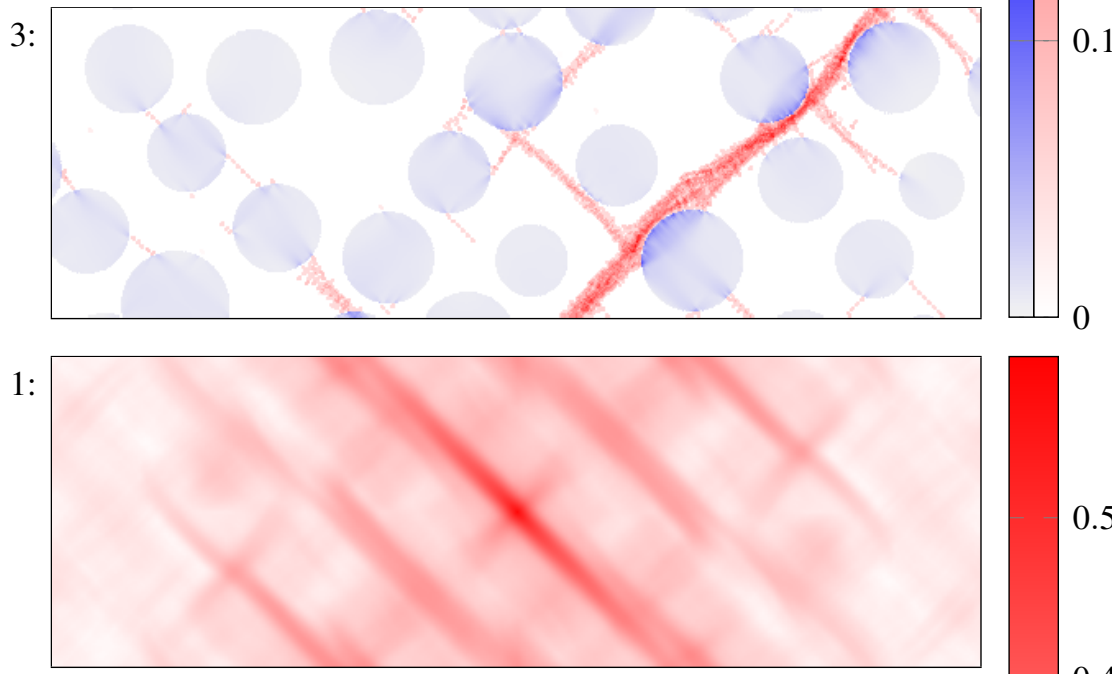

2:

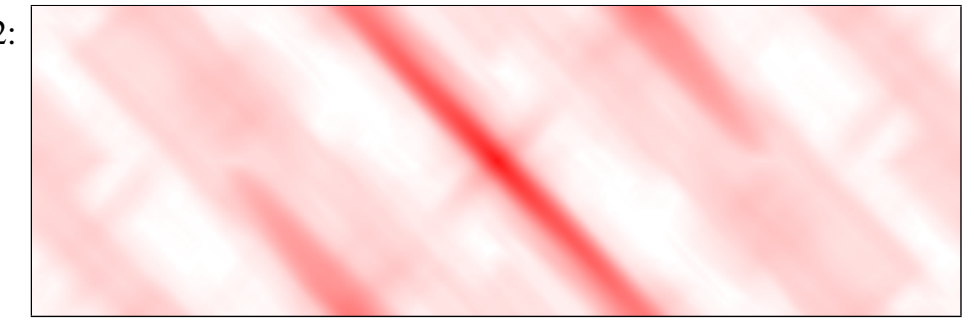

0.4

3:

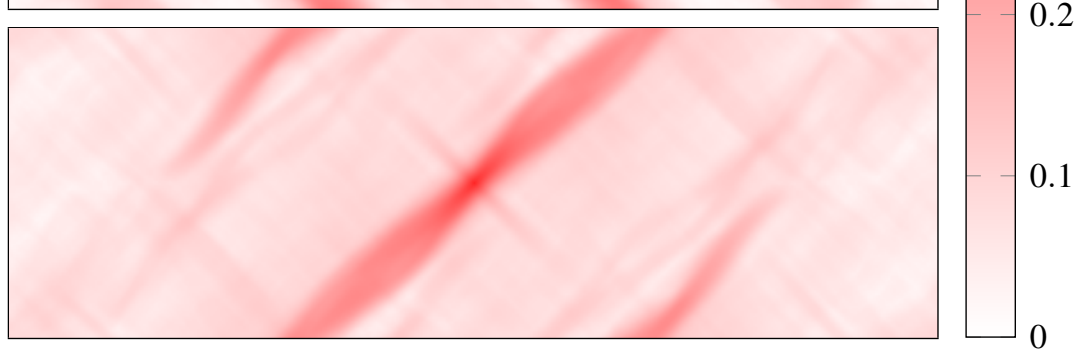

Figure C.4: Strain and two-point localization plot for $v=40, l=30, r=50$. 


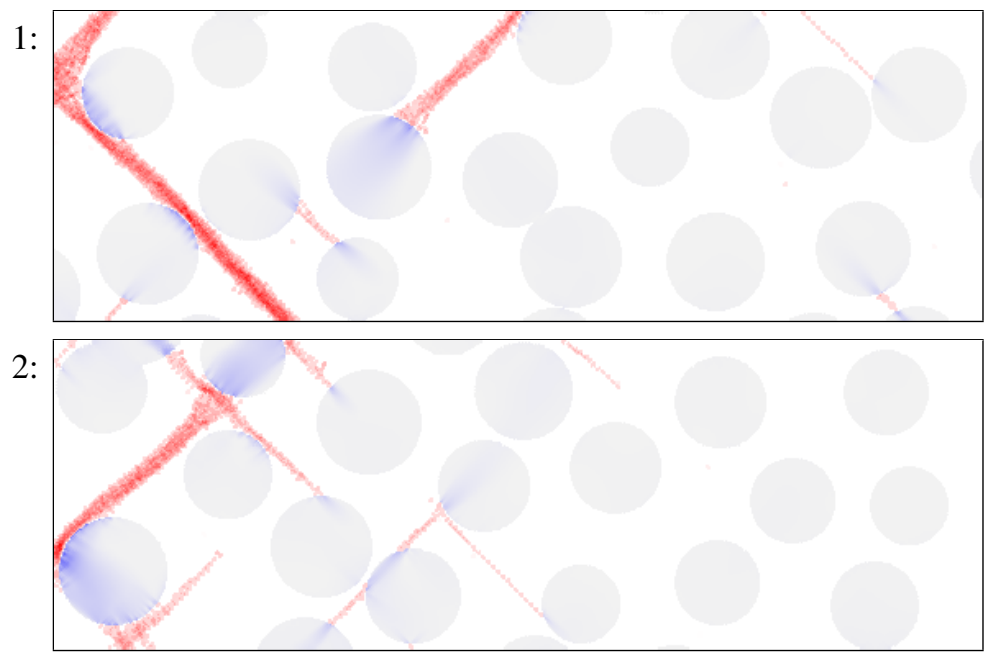

3:
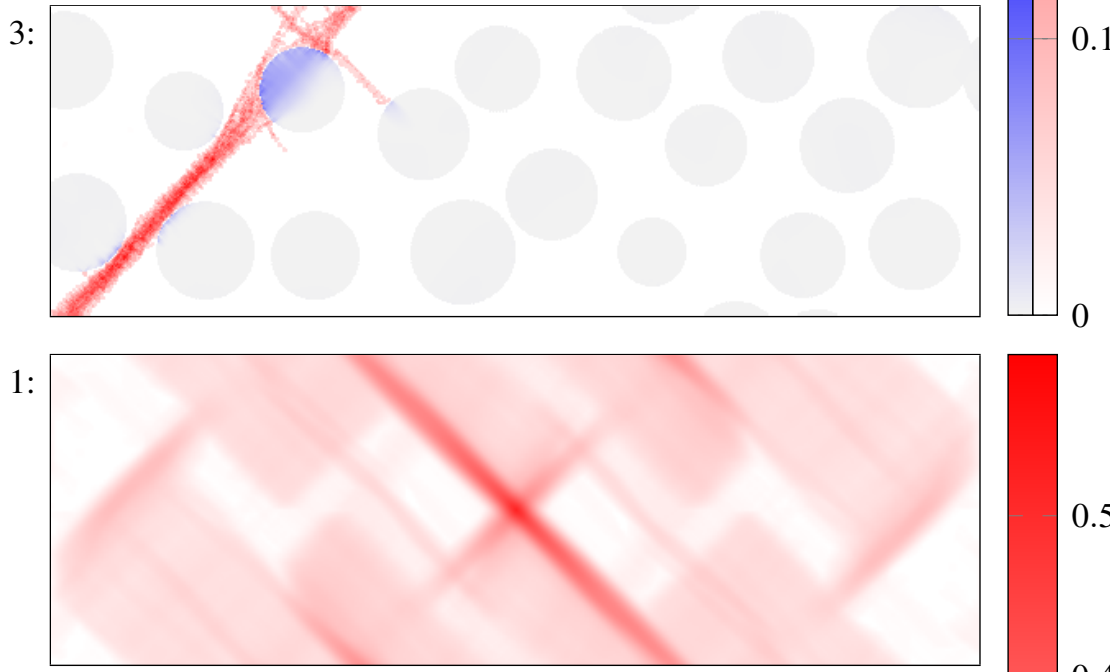

2:

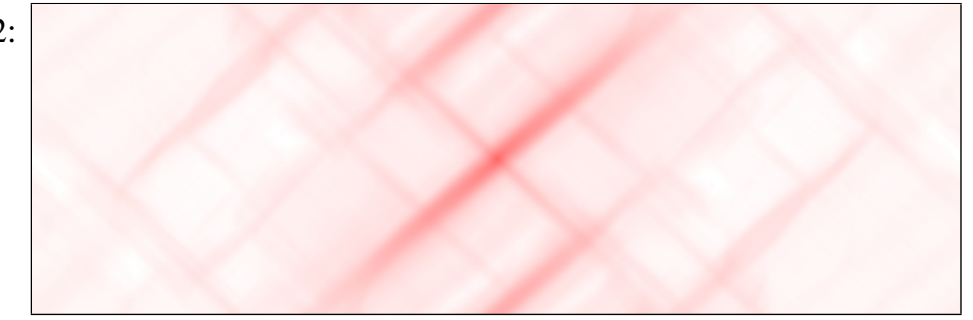

3:

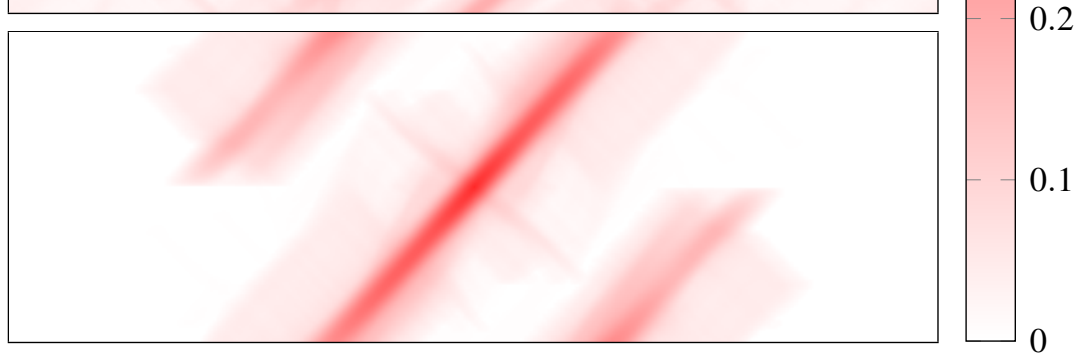

Figure C.5: Strain and two-point localization plot for $v=40, l=30, r=80$. 


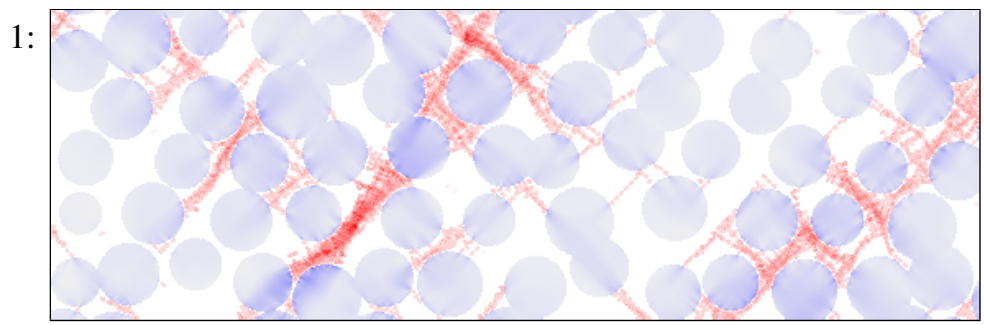

2:

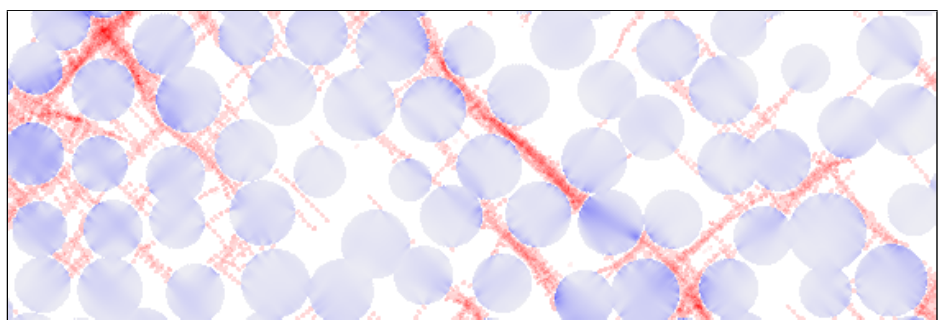

3:

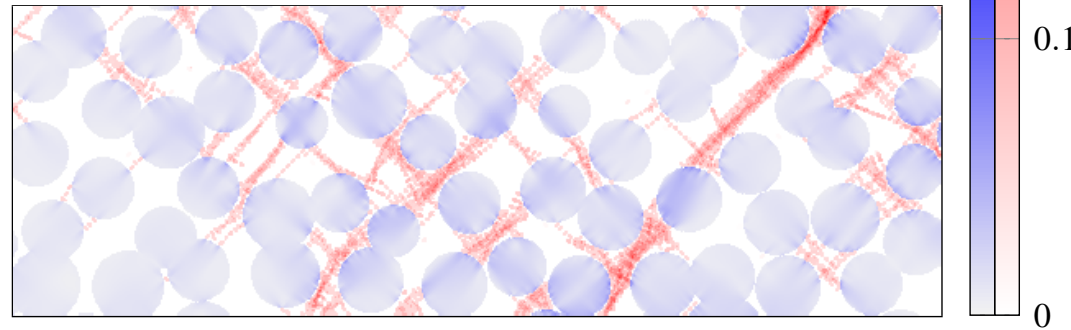

1 :

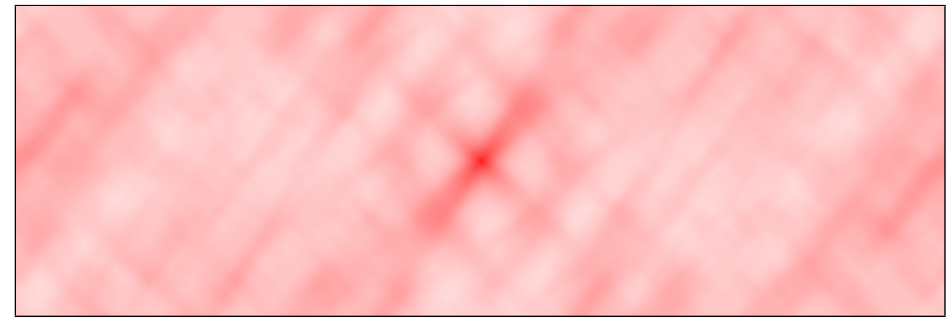

2:

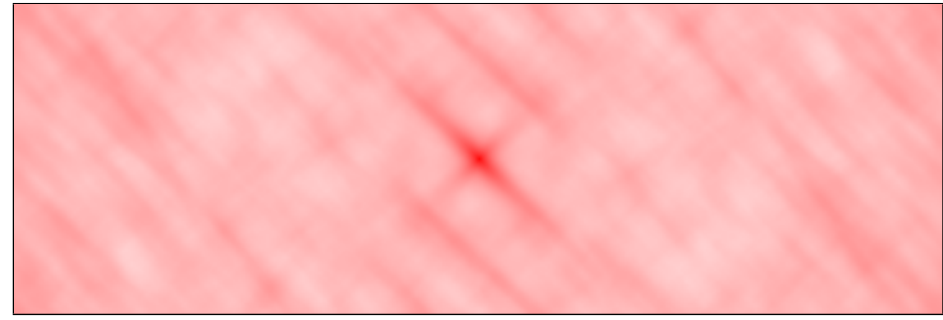

3:

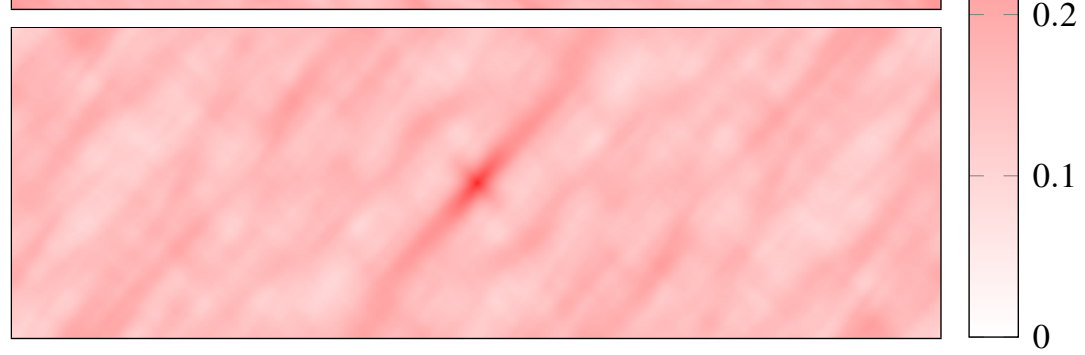

Figure C.6: Strain and two-point localization plot for $v=60, l=20, r=50$. 


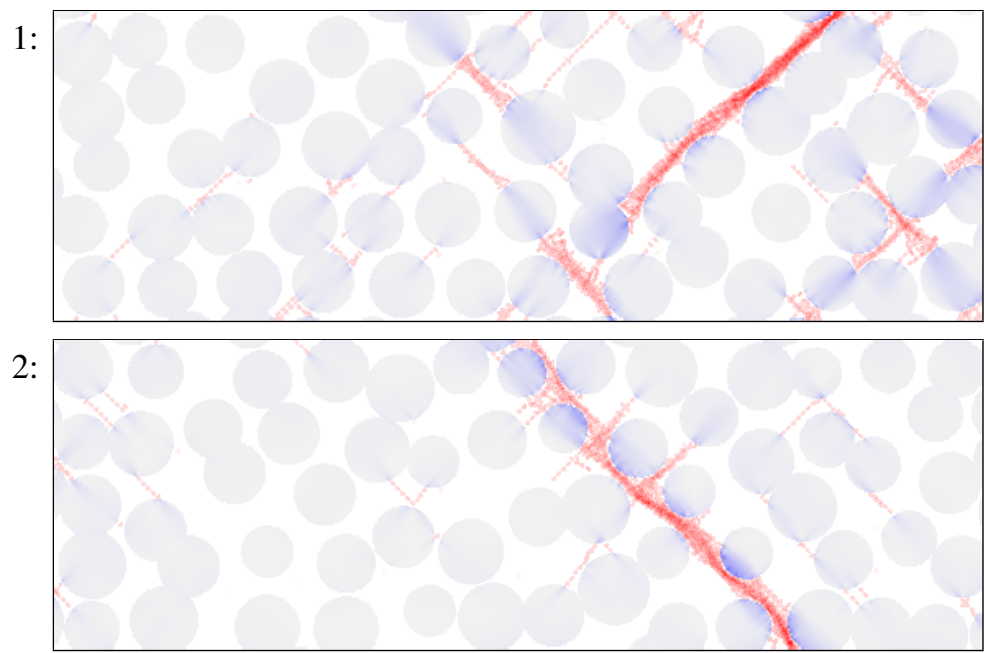

3:
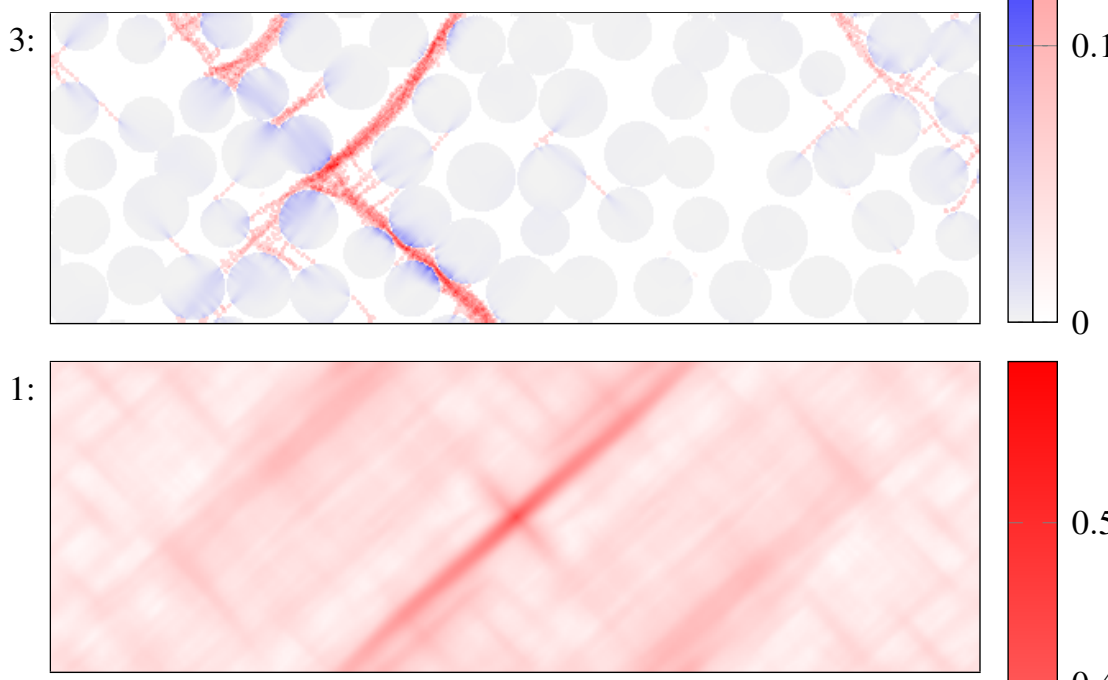

2:
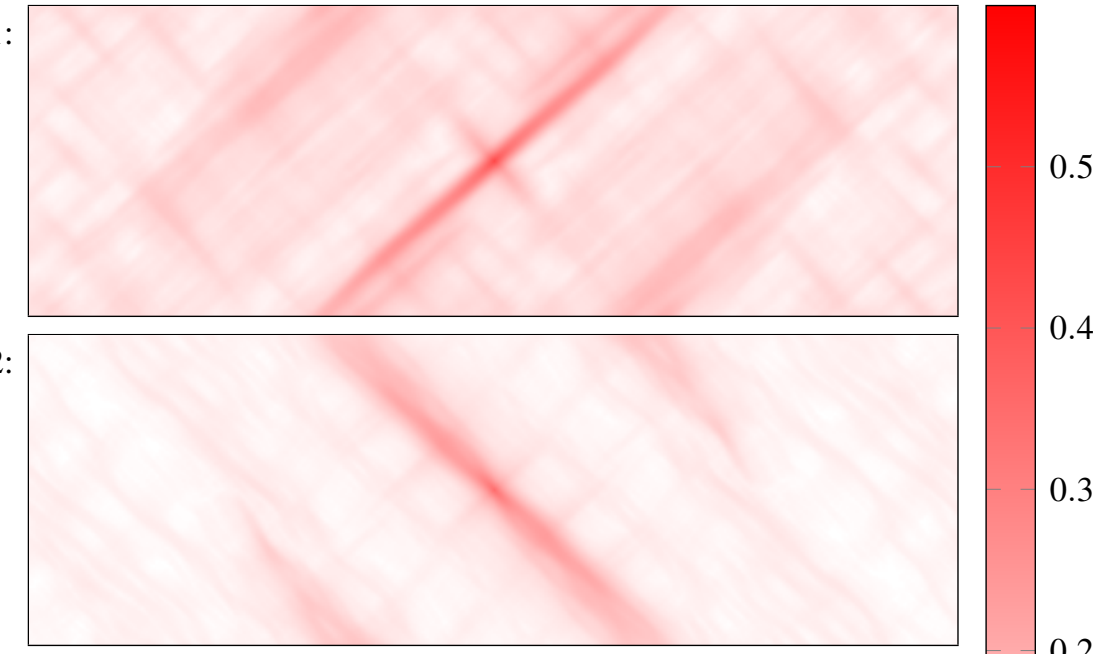

3:

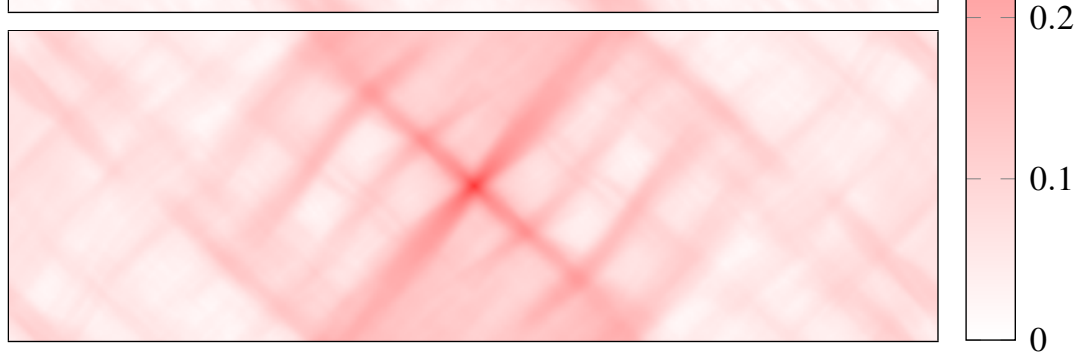

Figure C.7: Strain and two-point localization plot for $v=60, l=20, r=80$. 


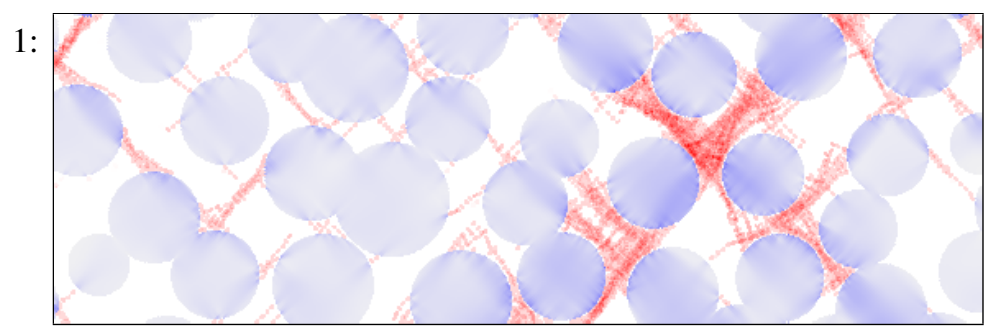

2:

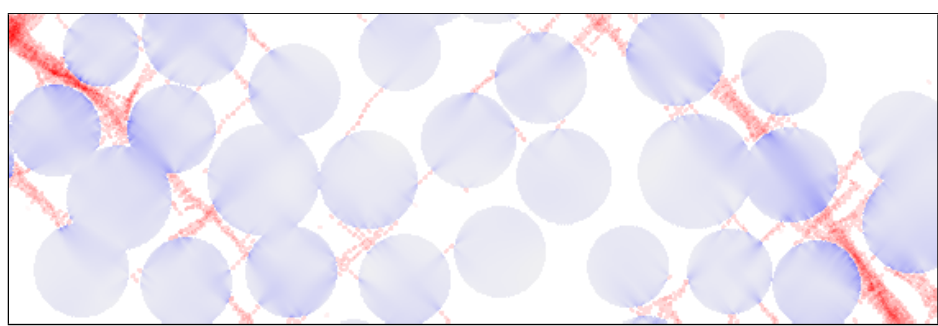

3:

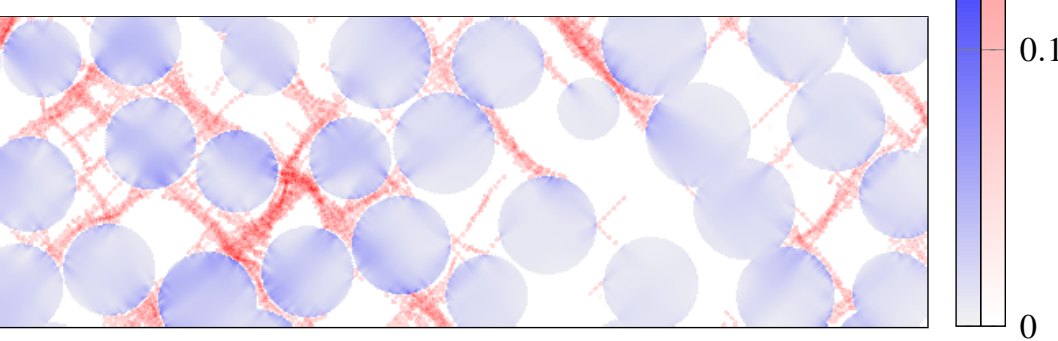

1 :

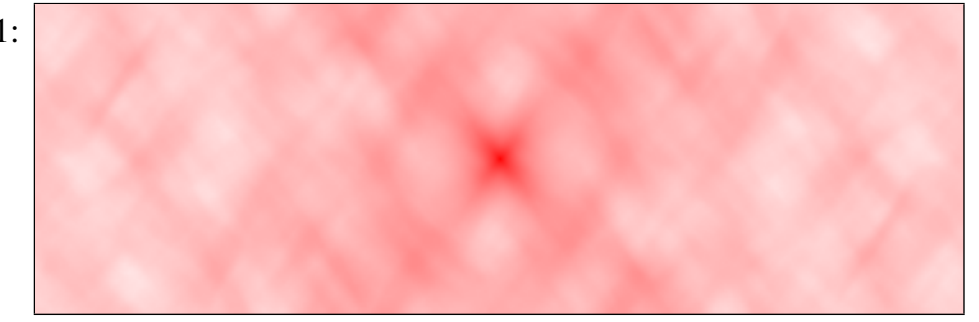

2:

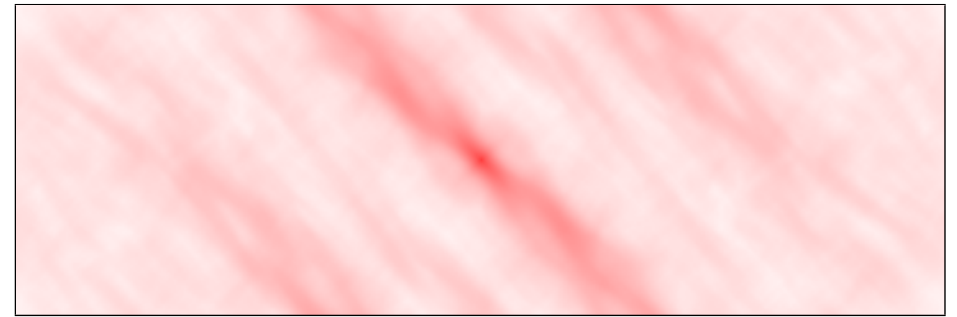

3:

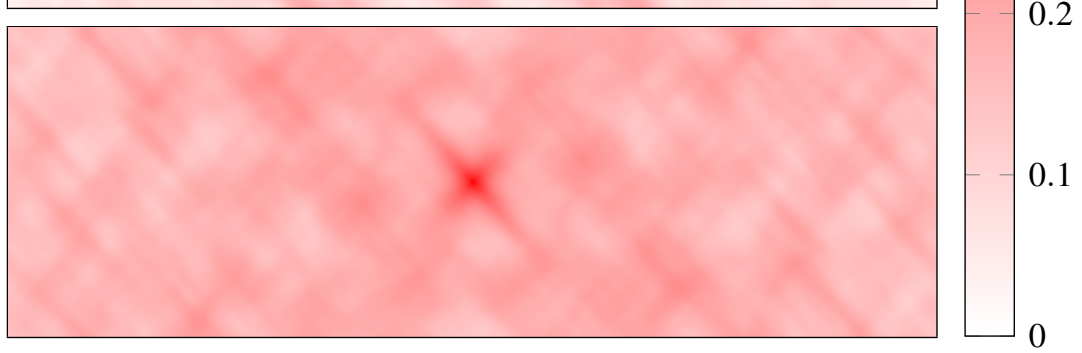

Figure C.8: Strain and two-point localization plot for $v=60, l=30, r=50$. 
1:

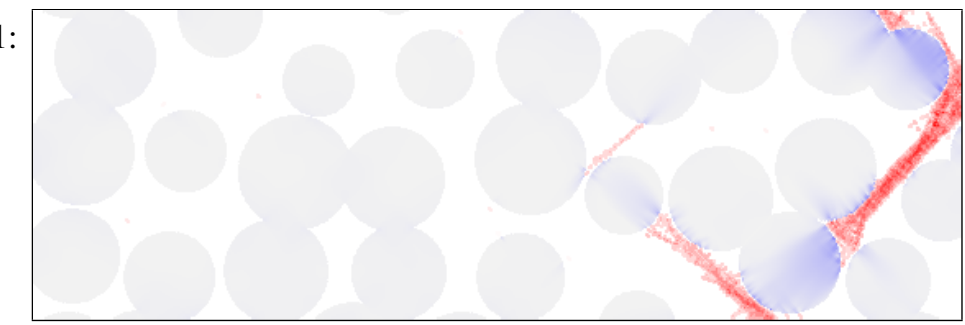

2:

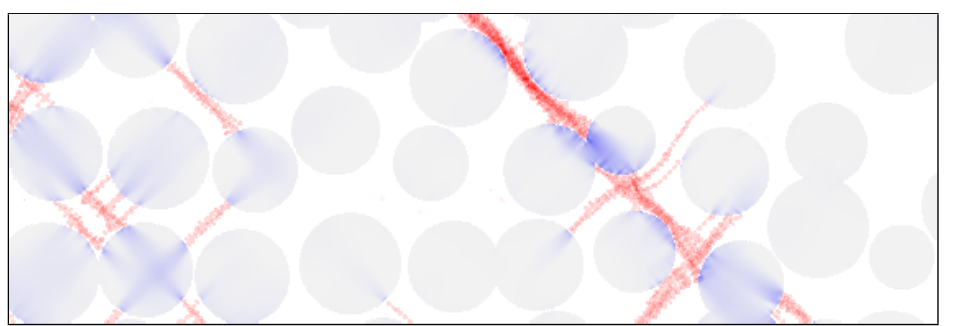

3:
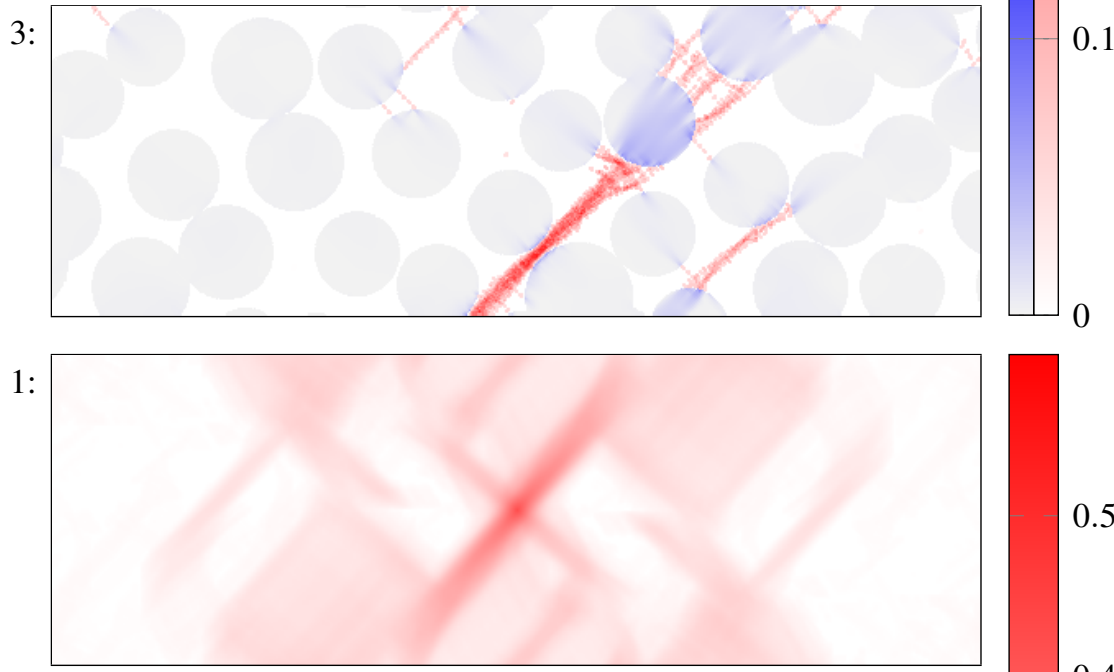

2:

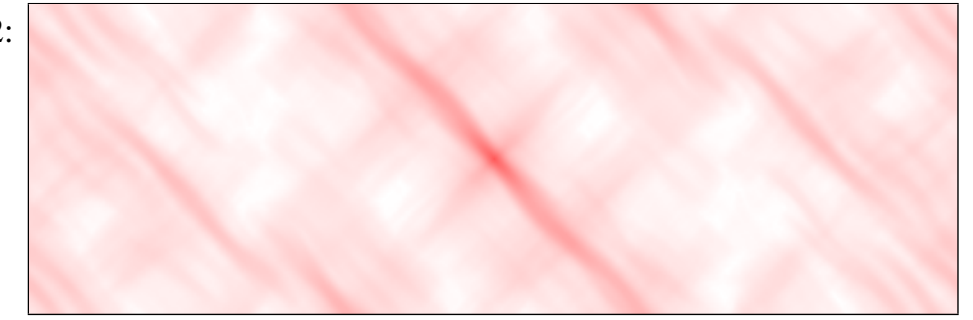

3:

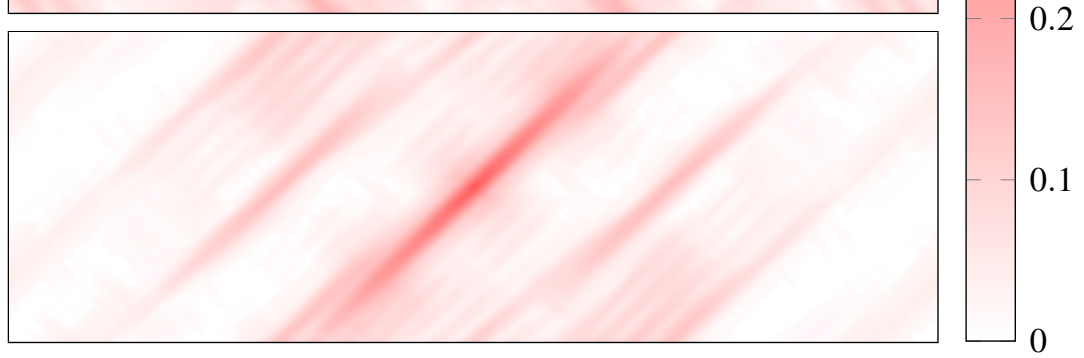

Figure C.9: Strain and two-point localization plot for $v=60, l=30, r=80$. 


\section{APPENDIX D. REGRESSION ANALYSIS MATHEMATICA CODE}

\section{ClearAll ["Global “*”];}

SetDirectory [ NotebookDirectory []];

Labels=Import ["Stat6 . mat","Labels"]// Quiet ;

Stat6=Import ["Stat 6 . mat"]// Quiet ;

$X=S$ tat 6 [ [ Position [Labels, ,X"][[1,1]]]];

Y=Stat 6 [[ Position [Labels,"Y"][[1,1]]]];

$\mathrm{YP}=$ Stat 6 [[ Position [Labels ,"YP"][[1,1]]]];

DepVarLabels=Stat 6 [ [ Position [Labels ," DepVarLabels "] [ [1,1]]]]//

Flatten;

IndVarLabels=Stat6 [[ Position [Labels ," IndVarLabels "] [ [1,1]]]]//

Flatten

$\mathrm{XC}=\mathrm{Table}[(\mathrm{X}[[\mathrm{i}, \mathrm{j}]]-(\operatorname{Max}[\mathrm{X}[[\mathrm{A} 11, \mathrm{j}]]]+\operatorname{Min}[\mathrm{X}[[\mathrm{A} 11, \mathrm{j}]]]) / 2) /((\operatorname{Max}[\mathrm{X}[[$

All, $\mathrm{j}]]]-\operatorname{Min}[X[[A 11, j]]]) / 2),\{i, 1, \operatorname{Length}[X]\},\{j, 1, \operatorname{Length}[X$ $[[1]]]\}]$;

MatrixForm [ Transpose [Transpose $[\mathrm{XC}]^{\sim}$ Join $^{\sim}$ Transpose $[\mathrm{X}]^{\sim}$ Join $\sim$ Transpose [Y]]]

MatrixForm [ Transpose [ Transpose $\left.[\mathrm{XC}]^{\sim} \mathrm{Join}^{\sim} \operatorname{Transpose}_{\mathrm{X}}\right]^{\sim} \mathrm{Join}^{\sim}$

Transpose [YP]] ]

$\operatorname{Map}[\operatorname{Max}, \operatorname{Transpose}[\operatorname{Abs}[\mathrm{Y}]]]^{\wedge}-1$

MatrixForm [ Transpose [ Transpose [XC] Join $\sim$ Transpose [YP]] ]

CenterPos=Flatten [ Position [Map[Norm,XC],0.0]]

CubePos=Complement $[$ Table $[\mathrm{i},\{\mathrm{i}, 1$, Length $[\mathrm{XC}]\}]$, CenterPos $]$

ExpMatCube $\left[i_{-}\right]:=$Transpose $\left[\right.$Transpose $[\mathrm{XC}[[\text { CubePos }]]]^{\sim}$ Join $\sim$ YP $[[$

CubePos, i ] ] \} 
ExpMatCenter $\left[i_{-}\right]:=$Transpose $\left[\right.$Transpose $[\mathrm{XC}[[\text { CenterPos }]]]^{\sim}$ Join $\sim\{$ YP $[[$ CenterPos, i] ] \} ]

ExpMatAll [ $\left.i_{-}\right]:=$ExpMatCube [ i ] Join $\sim$ ExpMatCenter [i ]

$\operatorname{Rnd}\left[\mathrm{x}_{-}, \mathrm{i}_{-}\right]:=\operatorname{Round}\left[\mathrm{x} * 10^{\wedge}(\mathrm{i}+1), 10\right] / 10.0^{\wedge}(\mathrm{i}+1)$

AnovaFullFit $\left[\mathrm{lm}_{-}, \mathrm{dep}_{-}\right]:=$Module $[\{$YPhat, YBar, SSE, SST, SSR , k, n, dfreg, dfre s , MSR, MSE, F, P, $1 \mathrm{mfn}\}$,

$\operatorname{lmfn}\left[\left\{\mathrm{a}_{-}, \mathrm{b}_{-}, \mathrm{c}_{-}\right\}\right]:=\operatorname{lm}[\mathrm{a}, \mathrm{b}, \mathrm{c}]$;

YPhat $=$ Map $[\operatorname{lmfn}, X]$;

YBar=Mean [YP [ [ A11, dep ] ] ];

SSE $=$ Total [( YPhat-YP [ [ All, dep ] ] )^2];

SST $=$ Total [(YP [ [ All, dep]]-YBar $\left.)^{\wedge} 2\right]$;

$\mathrm{SSR}=$ Total $\left[(\text { YPhat }- \text { YBar })^{\wedge} 2\right]$;

$\mathrm{k}=$ Length [ $\operatorname{lm}[$ " BasisFunctions"]] -1 ;

$\mathrm{n}=$ Leng th [ YPhat ];

$\mathrm{d}$ freg $=\mathrm{k}$;

$\mathrm{d}$ f r e s $=\mathrm{n}-\mathrm{k}-1$;

$\mathrm{MSR}=\mathrm{SSR} / \mathrm{d}$ freg ;

$\mathrm{MSE}=\mathrm{SSE} / \mathrm{d}$ fre s ;

$\mathrm{F}=\mathrm{MSR} / \mathrm{MSE}$;

Needs ["HypothesisTesting "”];

P= If [NumberQ [MSR], OneSidedPValue / . FRatioPValue [F, dfreg, dfres ] ,0 ];

$\{$ TableForm $[\{\{$ "Regression ", dfreg, SSR,MSR, F,P $\},\{$ "Error", dfres , SSE, MSE $\},\{$ "Total ",dfres+dfreg, SST $\}\}], P\}]$

AnovaFullFit $\left[1 \mathrm{~m}_{-}\right]:=\operatorname{Module}[\{$ Ydat, Yhat, YBar, SSE, SST, SSR, k, n, dfreg, dfre s , MSR, MSE, F, P, $1 \mathrm{mfn}\}$,

Ydat $=\operatorname{lm}[$ " Data"] [ [ A11, - 1]];

Yhat $=\operatorname{lm}[$ "PredictedResponse"];

YBar=Mean [ Ydat ];

SSE $=$ Total [( Yhat - Ydat $\left.)^{\wedge} 2\right]$;

$\mathrm{SST}=$ Total [( Ydat - YBar $\left.)^{\wedge} 2\right]$; 
$\mathrm{SSR}=$ Total $\left[(\text { Yhat }- \text { YBar })^{\wedge} 2\right]$;

k= If [MemberQ[ImRoM[" BasisFunctions"], 1], Length [1m [" BasisFunctions

"]] -1 , Length [ Im [" BasisFunctions"] ]];

n=Length [ Yhat ];

dfreg $=\mathrm{k}$;

$\mathrm{d}$ fres $=\mathrm{n}-\mathrm{k}-1$;

$\mathrm{MSR}=\mathrm{SSR} / \mathrm{d}$ freg ;

$\mathrm{MSE}=\mathrm{SSE} / \mathrm{d}$ fre s ;

$\mathrm{F}=\mathrm{MSR} / \mathrm{MSE}$;

Needs ["Hypothesis Testing “"];

P=Rnd [ If [ NumberQ[MSR], OneSidedPValue /. FRatioPValue [F, dfreg, dfres ], 0$], 4]$;

$\{$ TableForm $[\{\{$ "Regression”, dfreg, SSR, MSR, F, P $\},\{$ Error”, dfres , SSE, $\operatorname{MSE}\},\{"$ Total",dfres +dfreg, SST $\}\}], P\}]$

OutTabFn $\left[\operatorname{lmReg}_{-}, \operatorname{dep}_{-}\right]:=$Module $[\{$PresentVars , UniqueXC, UniqueMeanY,

RegXC, RegYhat, OutTab , ImRegFn, GradVect \},

Unique $\mathrm{XC}=\mathrm{Union}[\mathrm{XC}]$;

UniqueMeanYP=Table [Mean [YP [ [ Flatten [ Position [XC, UniqueXC [[i ] ] ] , dep ] ] , $\{$ i , 1, Length [UniqueXC ] $\}$;

$\operatorname{RegXC}=$ UniqueXC ;

PresentVars=Union@Cases [ $\operatorname{lmReg}[$ ” BestFit”], Except [ _-Symbol?(

Context@\#==="System “"\&),_Symbol],\{1, Infinity $\}$ ];

$\operatorname{RegXC}[[\mathrm{A} 11,1]]=\operatorname{If}[$ MemberQ $[$ PresentVars ,V] $, \operatorname{RegXC}[[\mathrm{A} 11,1]], \operatorname{RegXC}[[$

$\mathrm{A} 11,1]] * 0]$;

$\operatorname{RegXC}[[$ Al1 ,2 ] ] = If [MemberQ [PresentVars ,L], $\operatorname{RegXC}[[$ A11 ,2 ] ] $\operatorname{RegXC}[[$

$\mathrm{A} 11,2]] * 0]$;

$\operatorname{RegXC}[[$ All , 3 ] ] = If [MemberQ[PresentVars , R] , RegXC [[ A11, 3]], RegXC [ [

$\mathrm{A} 11,3]$ ] $* 0]$;

$\operatorname{RegXC}=U$ nion $[\operatorname{RegXC}]$;

$\operatorname{lm} \operatorname{RegFn}\left[\left\{\mathrm{a}_{-}, \mathrm{b}_{-}, \mathrm{c}_{-}\right\}\right]:=\operatorname{lm} \operatorname{Reg}[\mathrm{a}, \mathrm{b}, \mathrm{c}]$; 
Reg YPhat=Map [ $\operatorname{lmRegFn~,~RegXC~];~}$

GradVect $=$ Table $[\mathrm{D}[\operatorname{lmRegFn}[\{\mathrm{V}, \mathrm{L}, \mathrm{R}\}], \mathrm{v}],\{\mathrm{v},\{\mathrm{V}, \mathrm{L}, \mathrm{R}\}\}] / . \mathrm{V}->0 / . \mathrm{L}->0 / . \mathrm{R}$ $->0$

GradVect $=$ If [Norm [ GradVect $] !=0.0,0.80 *$ GradVect $/$ Norm [ GradVect ],

GradVect ];

OutTab $=$ Transpose [UniqueXC ] Join $\sim\{$ UniqueMeanYP $\} \sim$ Join $\sim$ Transpose [

$\operatorname{RegXC}]^{\sim}$ Join $\sim\{\operatorname{Reg} Y$ Phat $\} \sim \operatorname{Join} \sim$ Table $[\{-\operatorname{GradVect}[[\mathrm{q}]]\},\{\mathrm{q}, 1,3\}]^{\sim}$

Join $\sim$ Table $[\{2 * \operatorname{GradVect}[[\mathrm{q}]]\},\{\mathrm{q}, 1,3\}]$;

OutTab=OutTab Flatten $\sim\{2\}$;

Export ["ExpTab"<> ToString [dep] $<>$ ".txt",OutTab,"Table"];

OutTab // TableForm ]

$\mathrm{n}=2$;

AnovaFns $=\operatorname{Sum}\left[\left(\mathrm{V}^{\wedge} \mathrm{i}\right) *\left(\mathrm{~L}^{\wedge} \mathrm{j}\right) *\left(\mathrm{R}^{\wedge} \mathrm{k}\right),\{\mathrm{i}, 0, \mathrm{n}\},\{\mathrm{j}, 0, \mathrm{n}-\mathrm{i}\},\{\mathrm{k}, 0, \mathrm{n}-\mathrm{i}-\mathrm{j}\}\right]$;

AnovaFns=MonomialList $[$ AnovaFns, $\{\mathrm{V}, \mathrm{L}, \mathrm{R}\}$, ”

NegativeDegreeLexicographic" $]^{\sim} J o i n \sim\{V L R\}$

Clear $[\mathrm{n}]$

AllSubsets $=$ Subsets $[$ AnovaFns $[[2 ; ;-1]]]$;

AllSubsets $=$ Table $\left[\{1\}^{\sim}\right.$ Join $\sim$ AllSubsets [[i $\left.]\right],\{$ i, 1, Length [ AllSubsets

] \} ]

Alpha $=0.01$;

Analyze $\left[\operatorname{dep}_{-}\right]:=$Module $[\{$LinModels, LinModelsPMax, ValidLinModelPos,

ValidLinModels, LinModelsFullP, LinModelsSort, GradVect, lmRegFn \}, LinModels=Table [ Quiet [ LinearMode1Fit [ExpMatAll [dep ], AllSubsets [ [ i

]], $\{\mathrm{V}, \mathrm{L}, \mathrm{R}\}]],\{\mathrm{i}, 1$, Length [AllSubsets ] $\}]$;

LinModelsPMax=Table [Max[LinModels [[ i ] ] "ParameterPValues"] ], $\{$ i , 1,

Length [LinModels ] \} ];

ValidLin ModelPos=Flatten [ Position [LinModelsPMax, _?(\#<Alpha\&)] ];

ValidLinModels=LinModels [ [ ValidLinModelPos ] ];

LinModelsFullP=Table [ AnovaFullFit [ValidLinModels [[i ] ], dep ][[2]], \{

i , 1, Length [ValidLinModels ] \}]// Quiet ; 
ValidLinModelPos=Flatten [ Position [ LinModelsFullP, _? $(\#<$ Alpha\&)] ]

ValidLinModels=ValidLinModels [ [ ValidLinModelPos ] ];

LinModelsSort=Table [\{ValidLinModels [[i ] ] " AdjustedRSquared"],

ValidLinModels [[i ] ] $\},\{$ i , 1, Length [ValidLinModels ] $\}$;

LinModelsSort=Sort [ LinModelsSort ];

$\operatorname{lmReg}=$ LinModelsSort [[ - 1]][[2]];

$\operatorname{lm} \operatorname{RegFn}\left[\left\{\mathrm{a}_{-}, \mathrm{b}_{-}, \mathrm{c}_{-}\right\}\right]:=\operatorname{lm} \operatorname{Reg}[\mathrm{a}, \mathrm{b}, \mathrm{c}]$;

\{DepVarLabels [[ dep ] ] , ImReg ["BasisFunctions "], ImReg ["

ParameterTable"], AnovaFullFit [lmReg, dep][[1]], \{"s", Sqrt [lmReg

["EstimatedVariance "]] $\},\{$ "RSquared ", ImReg["RSquared "] $\},\{$,

AdjRSquared", ImReg [" AdjustedRSquared"] \}, OutTabFn[1mReg, dep ] \}//

MatrixForm ]

Analyze [1]

Table [Analyze [i ] , $\{$ i , 1, Length [ DepVarLabels ] $\}$ ]

$\operatorname{dep}=4$

$\mathrm{y}=\mathrm{Y}[[\mathrm{A} 11, \mathrm{dep}]]$

ExpMat=Transpose $\left[\right.$ Transpose $[X]^{\sim}$ Join $\left.\sim\{y\}\right]$;

ExpMat $[[$ All , 1] ] = ExpMat [ [ A11, 1] ] $/ 100$;

ExpMat [ [ All ,3] ] =xpMat [ [ A11 ,3 ] ]/100;

ExpMat// MatrixForm

RoMFns $=\{(\mathrm{r} * \mathrm{v}),(1-\mathrm{v})\}$

ImRoM=Line arMode1Fit [ExpMat, RoMFns, $\{\mathrm{v}, 1, \mathrm{r}\}$, IncludeConstant Basis $\rightarrow$

False ]

$\operatorname{lmRoM}[$ "RSquared "]

$\operatorname{lmRoM}["$ ParameterTable "]

AnovaFullFit [ $1 \mathrm{mRoM}]$ 